\title{
Dynamic Analysis on a Diffusive Two-Enterprise Interaction Model with Two Delays
}

\author{
Yanxia Zhang (iD) and Long Li (iD) \\ School of Mathematics and Information Engineering, Chongqing University of Education, Chongqing 400065, China \\ Correspondence should be addressed to Yanxia Zhang; zhangyx@cque.edu.cn
}

Received 15 November 2021; Revised 8 January 2022; Accepted 12 January 2022; Published 24 February 2022

Academic Editor: Antonio Di Crescenzo

Copyright ( 2022 Yanxia Zhang and Long Li. This is an open access article distributed under the Creative Commons Attribution License, which permits unrestricted use, distribution, and reproduction in any medium, provided the original work is properly cited.

\begin{abstract}
The oscillation and instability of systems caused by time delays have been widely studied over the past several decades. In nature, the phenomenon of diffusion is universal. Therefore, it is necessary to investigate the dynamic behavior of reaction-diffusion systems with time delays. In this study, a two-enterprise interaction model with diffusion and delay effects is considered. By analyzing the distribution of the roots of the corresponding characteristic equation, some conditions for the stability of the unique positive equilibrium and the existence of Hopf bifurcation at the steady state are investigated. As the sum of the time delays changes, there are a series of periodic solutions at the trivial steady-state solution of the system. In addition, the direction of Hopf bifurcation and the stability of the periodic solutions are discussed by using the normal form theory and the center manifold reduction of partial functional differential equations. Finally, numerical simulation experiments are conducted to illustrate the validity of the theoretical conclusions.
\end{abstract}

\section{Introduction}

As we all know, it is of great significance for an enterprise to consider its developmental progress and growth trend, grasp its position, and comprehensively analyze its interactive relationships with other enterprises in the business ecosystem. In recent years, the dynamic relationships among enterprises described by corresponding mathematical models have been widely studied [1-6]. In [5], Xu proposed the following model for the dynamic competition and cooperation between two enterprises and applied coincidence degree theory to analyze the existence of periodic solutions:

$$
\left\{\begin{array}{l}
\dot{x}_{1}(t)=r_{1} x_{1}(t)\left[1-\frac{x_{1}(t)}{K_{1}}-\frac{\alpha\left(x_{2}(t)-c_{2}\right)^{2}}{K_{2}}\right], \\
\dot{x}_{2}(t)=r_{2} x_{2}(t)\left[1-\frac{x_{2}(t)}{K_{2}}+\frac{\beta\left(x_{1}(t)-c_{1}\right)^{2}}{K_{1}}\right], \\
x_{1}(0) \geq 0, x_{2}(0) \geq 0,
\end{array}\right.
$$

where $x_{i}(t)(i=1,2)$ denotes the output of two enterprises, respectively; $r_{i}(i=1,2)$ represents the intrinsic growth rate of two enterprises, respectively; $K_{1}$ and $K_{2}$ are the carrying capacities of the natural market, i.e., load capacities of the two enterprises under unrestricted conditions; $\alpha$ is the consumption coefficient from the enterprise with output $x_{2}(t)$ to the one with output $x_{1}(t) ; \beta$ is the transformation coefficient from the enterprise with output $x_{1}(t)$ to the one with output $x_{2}(t)$; and $c_{i}(i=1,2)$ represents the initial output of the two enterprises, respectively. It was found that the outputs of the two enterprises reach an equilibrium state and at least have a positive periodic solution when the intrinsic growth rates, natural market loads, competition coefficients, and initial productions of the two enterprises meet certain conditions. Considering that the real dynamic ecological system always depends on information from the historical system, Liao et al. [3, 4] proposed two different competition and cooperation models for two enterprises with a delay (or delays) in succession. They investigated the effect of the delay (or delays) on the stability of the positive equilibrium by choosing $\tau$ (or $\tau_{1}, \tau_{2}$ ) as the bifurcation 
parameter (or parameters). On the basis of [3], Guerrini [1] further proposed that when the two delays are small, an approximate, simple method can be used to obtain the roots of the characteristic equation. An analysis of the dynamic behavior of a competition and cooperation model for two enterprises with two small delays was also provided. Considering the influence of periodic solutions on the development and operation of enterprises, $\mathrm{Xu}$ and $\mathrm{Li}$ [6] investigated almost periodic solutions for a competition and cooperation model of two enterprises with time-varying delays and feedback controls. The existence and global stability of the almost periodic solutions were obtained by establishing a suitable Lyapunov functional. In fact, the internal and external effects of time delays were found to be completely different for enterprises. Hence, Li et al. [2] proposed the following differential equations model with four different delays:

$$
\left\{\begin{array}{l}
\dot{x}_{1}(t)=r_{1} x_{1}(t)\left[1-\frac{x_{1}\left(t-\tau_{1}\right)}{K_{1}}-\frac{\alpha\left(x_{2}\left(t-\tau_{2}\right)-c_{2}\right)^{2}}{K_{2}}\right], \\
\dot{x}_{2}(t)=r_{2} x_{2}(t)\left[1-\frac{x_{2}\left(t-\tau_{3}\right)}{K_{2}}+\frac{\beta\left(x_{1}\left(t-\tau_{4}\right)-c_{1}\right)^{2}}{K_{1}}\right], \\
x_{1}(t)=\varphi(t), x_{2}(t)=\psi(t), t \in\left[-\max _{1 \leq i \leq 4}\left\{\tau_{i}\right\}, 0\right],
\end{array}\right.
$$

where $\varphi(t), \psi(t) \in C\left(\left[-\max _{1 \leq i \leq 4}\left\{\tau_{i}\right\}, 0\right], \mathbb{R}\right), \quad$ and $\quad \tau_{i} \geq 0$ $(1 \leq i \leq 4)$ represent the delay effects within and between enterprises, respectively. Specifically, $\tau_{1}$ and $\tau_{3}$ denote the gestation periods of the outputs for the two enterprises. $\tau_{2}$ and $\tau_{4}$ represent the block delay and promoting delay, respectively, imposed by one enterprise on another. One case where $\tau_{i}=\tau(1 \leq i \leq 4)$ in (2) was considered in [4], another case where $\tau_{3}=\tau_{1}, \tau_{4}=\tau_{2}$ was discussed in [3], and some corresponding results were obtained. In [2], Li et al. researched the stability of the positive equilibrium and the existence of Hopf bifurcation, deduced the formula determining the direction of Hopf bifurcation, and achieved the stability of bifurcating periodic solutions by setting $\tau_{1}=\tau_{3}=$ 0 and choosing $\tau=\tau_{2}+\tau_{4}>0$ as the parameter. It has shown that time delay has an important impact on the steady-state equilibrium of the interactive relationship between enterprises. The latest research results of some delayed systems can be found in [7-13], which focus on multiple time delays and feedback control, fractional-order delay model, and discrete model with multiple delays.

However, most of these studies only considered the effect of time delays and lost sight of the effect of spatial diffusion. It is well known that diffusion factors are indispensable in the modeling of ecological and biological systems and often utilized to describe the spatial distributions of the densities of some certain substances, such as plants, animals, and other organisms. At present, there are a growing number of dynamic models described by reaction-diffusion equations with time delays in various application areas [14-27]. For example, some scholars [19, 23, 25-27] proposed several different diffusive predator-prey systems with delays to analyze the effect of diffusion on biological systems. Huang et al. [18] researched a plant-pollinator model with diffusion and time delay effects and discussed its bifurcation and temporal periodic patterns. Some scholars $[16,22]$ established delayed reaction-diffusion systems to investigate the spatiotemporal transmission of bacteria and viruses in epidemiology. As a result of the impact of the growth pole on the economic activities of industrial groups, $\mathrm{Hu}$ et al. [17] provided a Kaldor-Kalecki model of the business cycle with diffusion and time delay and described the influence of the diffusion coefficient on the spatial pattern of the system.

Generally, in the development of the regional economy, the enterprises located in the center of the city have the functions of production, information, transportation, trade, service, and decision-making, which act like a "magnetic pole" that attracts production from the surrounding areas and spreads its own production factors to the surrounding areas. Especially when the industrial economic development in the central area is at the mature stage, the diffusion effect continues to increase. Therefore, based on the above argument, we further consider spatial diffusion with zero-flux boundary conditions. A delayed reaction-diffusion system for a two-enterprise interaction should be described by the following model:

$$
\begin{cases}\frac{\partial x_{1}}{\partial t}=D_{1} \Delta x_{1}+r_{1} x_{1}(t, x)\left[1-\frac{x_{1}(t, x)}{K_{1}}-\frac{\alpha\left(x_{2}\left(t-\tau_{2}, x\right)-c_{2}\right)^{2}}{K_{2}}\right], & x \in \Omega, t>0, \\ \frac{\partial x_{2}}{\partial t}=D_{2} \Delta x_{2}+r_{2} x_{2}(t, x)\left[1-\frac{x_{2}(t, x)}{K_{2}}+\frac{\beta\left(x_{1}\left(t-\tau_{1}, x\right)-c_{1}\right)^{2}}{K_{1}}\right], & x \in \Omega, t>0, \\ \frac{\partial x_{1}}{\partial v}=\frac{\partial x_{2}}{\partial v}=0, \quad t \geq 0, x \in \partial \Omega, & \\ x_{1}(t, x)=\varphi(t, x) \geq 0, x_{2}(t, x)=\psi(t, x) \geq 0,(t, x) \in\left[-\max _{1 \leq i \leq 2}\left\{\tau_{i}\right\}, 0\right] \times \bar{\Omega},\end{cases}
$$


where $x_{i}(t, x)(i=1,2)$ denotes the output of two enterprises at time $t$ and spatial position $x$, respectively. $x_{i}\left(t-\tau_{i}, x\right)$ $(i=1,2)$ represents the output of two enterprises in the gestation period at spatial state $x$, respectively. $\left(1-x_{i}(t, x)\right) / K_{i}(i=1,2)$ reflects the prevention effect on the output of enterprise $i(i=1,2)$ caused by its own consumption of limited resources, respectively. As the two enterprises exist in the same environment, the impact of the delay, caused by enterprise 2 consuming the same limited resources in the process of development, on enterprise 1 is expressed by $-\left(\alpha\left(x_{2}\left(t-\tau_{2}, x\right)-c_{2}\right)^{2}\right) / K_{2}$, and the promoting delay by which enterprise 1 supports enterprise 2 is denoted as $\beta\left(x_{1}\left(t-\tau_{1}, x\right)-c_{1}\right)^{2} / K_{1}$. In [3], it was suggested that the promoting delay and blocking delay in the gestation period of output for enterprises have important economic significance for their development through changing parameters $\alpha$ and $\beta . \Delta$ is the Laplacian operator, and $D_{1}, D_{2}>0$ denote the diffusion coefficients. $\Omega$ represents a bounded domain in $\mathbb{R}^{N}(N \geq 1)$ with a smooth boundary $\partial \Omega$, and $v$ is the outward unit normal vector on $\partial \Omega$. Similar to [2-4], by introducing the terms $\alpha_{i}=\left(r_{i} / K_{i}\right)(i=1,2), b_{1}=\left(\alpha r_{1} / K_{2}\right)$, $b_{2}=\left(\beta r_{2} / K_{1}\right), d_{i}=r_{i}-a_{i} c_{i}, y_{i}(t, x)=x_{i}(t, x)-c_{i}(i=1,2)$, we obtain the following model:

$$
\begin{cases}\frac{\partial y_{1}}{\partial t}=D_{1} \Delta y_{1}+\left(y_{1}(t, x)+c_{1}\right)\left[d_{1}-a_{1} y_{1}(t, x)-b_{1} y_{2}^{2}\left(t-\tau_{2}, x\right)\right], \quad x \in \Omega, t>0 \\ \frac{\partial y_{2}}{\partial t}=D_{2} \Delta y_{2}+\left(y_{2}(t, x)+c_{2}\right)\left[d_{2}-a_{2} y_{2}(t, x)+b_{2} y_{1}^{2}\left(t-\tau_{1}, x\right)\right], \quad x \in \Omega, t>0 \\ \frac{\partial y_{1}}{\partial v}=\frac{\partial y_{2}}{\partial v}=0, \quad t \geq 0, x \in \partial \Omega \\ y_{1}(t, x)=\varphi(t, x) \geq 0, y_{2}(t, x)=\psi(t, x) \geq 0,(t, x) \in\left[-\max _{1 \leq i \leq 2}\left\{\tau_{i}\right\}, 0\right] \times \bar{\Omega} .\end{cases}
$$

For simplicity, we reduce the spatial domain $\Omega$ to one dimension $(0, \pi)$. Although there is an abundant literature about the stability and Hopf bifurcation of delayed differential equation models with diffusion, stability analysis of the interactive behaviors among enterprises in the business ecosystem, especially reaction-diffusion financial systems with delays, is very rare. The main contributions of this study are as follows: first, the interaction between two enterprises is discussed from the perspective of ecology, and the factors such as multiple time delay and diffusion affecting the dynamic properties of the system are introduced into the model, which makes the given model more in line with the actual background in economics than the previous models. Second, the complex dynamic properties of the system are discussed, including the stability of the system and the qualitative analysis of the existence of Hopf bifurcation. The parameter calculation expressions of bifurcation direction, stability of bifurcation periodic solution, and period size are determined. Finally, we explain the results from the perspective of application and supplement the relevant conclusions of the existing literature.

The rest of this study is organized as follows. In Section 2, we consider the distribution of the roots of the characteristic equation associated linearized system and explore the stability of the positive steady state and the existence of Hopf bifurcation of system (4) to satisfy some requirements. In Section 3, according to the normal form method and the center manifold reduction developed by Hassard et al. [28], we determine the direction of Hopf bifurcation, give an explicit rule, and analyze the stability of bifurcating periodic solutions. In addition, some numerical simulations are conducted in Section 4 to verify our theoretical predictions. The conclusions are presented in Section 5.

\section{Stability and Hopf Bifurcation}

In this section, taking $\tau$ as the varying parameter, we analyze the distribution of the roots of the corresponding characteristic equation of (4) at the positive constant steady state and the stability of the positive equilibrium point. Some conditions of Hopf bifurcation of (4) are provided.

According to $[3,4]$, there is a unique positive constant steady state $E\left(y_{1}^{*}, y_{2}^{*}\right)$ of system (4), which provides that the following condition holds:

$$
\text { (H1): } a_{2}^{2} d_{1}>b_{1} d_{2}^{2}
$$

Let $\quad u_{1}(t, x)=y_{1}(t, x)-y_{1}^{*}, u_{2}(t, x)=y_{2}(t, x)-y_{2}^{*}$; then, (4) is equivalent to the following system: 


$$
\left\{\begin{array}{l}
\frac{\partial u_{1}(t, x)}{\partial t}=D_{1} \Delta u_{1}(t, x)+\left(u_{1}(t, x)+y_{1}^{*}+c_{1}\right)\left[d_{1}-a_{1}\left(u_{1}(t, x)+y_{1}^{*}\right)-b_{1}\left(u_{2}(t-\tau, x)+y_{2}^{*}\right)^{2}\right] \\
\frac{\partial u_{2}(t, x)}{\partial t}=D_{2} \Delta u_{2}(t, x)+\left(u_{2}(t, x)+y_{2}^{*}+c_{2}\right)\left[d_{2}-a_{2}\left(u_{2}(t, x)+y_{2}^{*}\right)+b_{2}\left(u_{1}(t, x)+y_{1}^{*}\right)^{2}\right] \\
\frac{\partial u_{1}}{\partial v}=\frac{\partial u_{2}}{\partial v}=0, \quad t \geq 0, x \in \partial \Omega \\
u_{1}(t, x)=\varphi(t, x)-y_{1}^{*}, u_{2}(t, x)=\psi(t, x)-y_{2}^{*},(t, x) \in\left[-\max _{1 \leq i \leq 2}\left\{\tau_{i}\right\}, 0\right] \times \bar{\Omega}
\end{array}\right.
$$

where $\tau=\tau_{1}+\tau_{2}$. (6) can be written as follows by setting $d_{1}-a_{1} y_{1}^{*}-b_{1}\left(y_{2}^{*}\right)^{2}=0$ and $d_{2}-a_{2} y_{2}^{*}+b_{2}\left(y_{1}^{*}\right)^{2}=0$.

$$
\left\{\begin{array}{l}
\frac{\partial u_{1}(t, x)}{\partial t}=D_{1} \Delta u_{1}(t, x)+\left(u_{1}(t, x)+y_{1}^{*}+c_{1}\right)\left[-a_{1} u_{1}(t, x)-b_{1} u_{2}^{2}(t-\tau, x)-2 b_{1} y_{2}^{*} u_{2}(t-\tau, x)\right] \\
\frac{\partial u_{2}(t, x)}{\partial t}=D_{2} \Delta u_{2}(t, x)+\left(u_{2}(t, x)+y_{2}^{*}+c_{2}\right)\left[-a_{2} u_{2}(t, x)+b_{2} u_{1}^{2}(t, x)+2 b_{2} y_{1}^{*} u_{1}(t, x)\right]
\end{array}\right.
$$

We assume that $\varphi, \psi \in \ell=C([-\tau, 0], X)$, and $X$ is defined by

$X=\left\{\left(u_{1}, u_{2}\right): u_{i} \in W^{2,2}(0, \pi), \frac{\partial u_{i}(t, x)}{\partial v}=0, i=1,2, x=0, \pi\right\}$,

with the inner product $\langle\cdot, \cdot\rangle$.

Denote $u_{i}(t)=u_{i}(t, \cdot)(i=1,2)$ and $U=\left(u_{1}, u_{2}\right)^{T}$. For $\phi=\left(\phi_{1}, \phi_{2}\right)^{T} \in \ell$, system (7) can be rewritten as the following abstract differential equation in the phase space $\ell=C([-\tau, 0], X)$ :

$$
\dot{U}(t)=D \Delta U(t)+L\left(U_{t}\right)+F\left(U_{t}\right),
$$

where $D=\operatorname{diag}\left(D_{1}, D_{2}\right)$ and $L: \ell \longrightarrow X, F: \ell \longrightarrow X$ are defined by the following:

$$
\begin{aligned}
& L(\phi)=\left(\begin{array}{cc}
A_{1} & 0 \\
A_{2} & B_{2}
\end{array}\right)\left(\begin{array}{l}
\phi_{1}(0) \\
\phi_{2}(0)
\end{array}\right)+\left(\begin{array}{cc}
0 & B_{1} \\
0 & 0
\end{array}\right)\left(\begin{array}{l}
\phi_{1}(-\tau) \\
\phi_{2}(-\tau)
\end{array}\right), \\
& F(\phi)=\left(\begin{array}{l}
F_{1}+F_{2} \\
F_{3}+F_{4}
\end{array}\right),
\end{aligned}
$$

where

$$
\begin{aligned}
& A_{1}=-a_{1}\left(y_{1}^{*}+c_{1}\right)<0, \\
& A_{2}=2 b_{2} y_{1}^{*}\left(y_{2}^{*}+c_{2}\right)>0, \\
& B_{1}=-2 b_{1} y_{2}^{*}\left(y_{1}^{*}+c_{1}\right)<0, \\
& B_{2}=-a_{2}\left(y_{2}^{*}+c_{2}\right)<0, \\
& F_{1}=-a_{1} \phi_{1}^{2}(0)-b_{1}\left(y_{1}^{*}+c_{1}\right) \phi_{2}^{2}(-\tau),
\end{aligned}
$$

$$
\begin{aligned}
F_{2} & =-2 b_{1} y_{2}^{*} \phi_{1}(0) \phi_{2}(-\tau)-b_{1} \phi_{1}(0) \phi_{2}^{2}(-\tau), F_{3} \\
& =-a_{2} \phi_{2}^{2}(0)+b_{2}\left(y_{2}^{*}+c_{2}\right) \phi_{1}^{2}(0), F_{4} \\
& =2 b_{2} y_{1}^{*} \phi_{2}(0) \phi_{1}(0)+b_{2} \phi_{2}(0) \phi_{1}^{2}(0), \phi(\theta) \\
& =\left(\phi_{1}(\theta), \phi_{2}(\theta)\right)^{T} \in \ell .
\end{aligned}
$$

It is easy to see that the linearization of (9) is given by

$$
\dot{U}(t)=D \Delta U(t)+L\left(U_{t}\right)
$$

and its characteristic equation is

$$
\lambda y-D \Delta y-L\left(e^{\lambda} y\right)=0
$$

where $y \in \operatorname{dom}\{\Delta\} \backslash\{0\}, \operatorname{dom}(\Delta) \subset X$.

According to the properties of the Laplacian operator on the bounded domain $[14,18]$, the operator $\Delta$ on $X$ has the eigenvalues $-k^{2}$ and the relative eigenfunctions $\beta_{k}^{1}=\left(\begin{array}{c}\cos k x \\ 0\end{array}\right), \beta_{k}^{2}=\left(\begin{array}{c}0 \\ \cos k x\end{array}\right)$, for $k \in \mathbb{N}_{0}=\{0,1,2, \cdots\}$. $\left(\beta_{k}^{1}, \beta_{k}^{2}\right)$ is a basis of the phase space $X$. So, any element $y \in X$ can be expanded to a Fourier series as follows:

$$
y=\sum_{k=0}^{\infty} Y_{k}^{T}\left(\begin{array}{l}
\beta_{k}^{1} \\
\beta_{k}^{2}
\end{array}\right)
$$

where $Y_{k}^{T}=\left(\left\langle y, \beta_{k}^{1}\right\rangle,\left\langle y, \beta_{k}^{2}\right\rangle\right)$.

By using some simple computations, we have

$$
L\left(\phi^{T}\left(\begin{array}{l}
\beta_{k}^{1} \\
\beta_{k}^{2}
\end{array}\right)\right)=L\left(\phi^{T}\right)\left(\begin{array}{c}
\beta_{k}^{1} \\
\beta_{k}^{2}
\end{array}\right), \quad k \in \mathbb{N}_{0}
$$


Hence, (13) is equivalent to

$$
\sum_{k=0}^{\infty} Y_{k}^{T}\left[\left(\lambda I_{2}+D k^{2}\right)-\left(\begin{array}{cc}
A_{1} & B_{1} e^{-\lambda \tau} \\
A_{2} & B_{2}
\end{array}\right)\right]\left(\begin{array}{l}
\beta_{k}^{1} \\
\beta_{k}^{2}
\end{array}\right)=0 .
$$

Therefore, all the characteristic roots of (13) are equivalent to the roots of the following equation:

$$
\begin{aligned}
\lambda^{2} & +\left(-A_{1}+D_{1} k^{2}-B_{2}+D_{2} k^{2}\right) \lambda+\left(-A_{1}+D_{1} k^{2}\right)\left(-B_{2}+D_{2} k^{2}\right) \\
& -A_{2} B_{1} e^{-\lambda \tau}=0 .
\end{aligned}
$$

Let $p=-\left(A_{1}+B_{2}\right)+D_{1} k^{2}+D_{2} k^{2}>0, q=-A_{2} B_{1}>0$, and $r=\left(-A_{1}+D_{1} k^{2}\right)\left(-B_{2}+D_{2} k^{2}\right)>0$; then, (17) can be rewritten as

$$
\lambda^{2}+p \lambda+r+q e^{-\lambda \tau}=0 .
$$

It is clear that $\lambda=0$ is not a root of (18) for $\forall k \in \mathbb{N}_{0}$. When $\tau=0$, (18) can be converted into

$$
\lambda^{2}+p \lambda+r+q=0 .
$$

Suppose $\lambda_{1}$ and $\lambda_{2}$ are two roots of (19). For $\forall k \in \mathbb{N}_{0}$, it is easy to see that

$$
\begin{aligned}
\lambda_{1}+\lambda_{2} & =-\left[-\left(A_{1}+B_{2}\right)+D_{1} k^{2}+D_{2} k^{2}\right]<0, \\
\lambda_{1} \lambda_{2} & =\left(-A_{1}+D_{1} k^{2}\right)\left(-B_{2}+D_{2} k^{2}\right)-A_{2} B_{1}>0 .
\end{aligned}
$$

Therefore, if (H1) holds, the unique positive equilibrium point $E\left(y_{1}^{*}, y_{2}^{*}\right)$ of (4) is asymptotically stable for $\tau=0$.

Furthermore, suppose that $\tau>0$ and $\lambda=i \omega(\omega>0)$ is a solution of (18). Then, $\omega$ satisfies the following equation for some $k \in \mathbb{N}_{0}$ :

$$
-\omega^{2}+i \omega p+r+q(\cos \omega \tau-i \sin \omega \tau)=0 .
$$
have

By separating the imaginary and real parts of (21), we

$$
\left\{\begin{array}{l}
\omega^{2}-r=q \cos \omega \tau, \\
p \omega=q \sin \omega \tau .
\end{array}\right.
$$

It follows from (22) that

$$
\omega^{4}+\left(p^{2}-2 r\right) \omega^{2}+r^{2}-q^{2}=0 .
$$

Let $z=\omega^{2}$; then, (23) becomes

$$
z^{2}+\left(p^{2}-2 r\right) z+r^{2}-q^{2}=0 .
$$

By performing some calculations, one can show that

$p^{2}-2 r=\left(-A_{1}+D_{1} k^{2}\right)^{2}+\left(-B_{2}+D_{2} k^{2}\right)^{2}$,

$r^{2}-q^{2}=\left(-A_{1}+D_{1} k^{2}\right)^{2}\left(-B_{2}+D_{2} k^{2}\right)^{2}-A_{2}^{2} B_{1}^{2}$.

Note that

$r+q=\left(-A_{1}+D_{1} k^{2}\right)\left(-B_{2}+D_{2} k^{2}\right)-A_{2} B_{1}>0$.

Hence, if the following condition holds,

$$
(\mathrm{H} 2): r-q=\left(-A_{1}+D_{1} k^{2}\right)\left(-B_{2}+D_{2} k^{2}\right)+A_{2} B_{1}>0,
$$

then (24) has no positive real roots, and the characteristic equation has no purely imaginary roots for all $\tau>0$. Therefore, we have the following conclusion if (H1) and (H2) hold.

Theorem 1. Assume that conditions (H1) and (H2) hold; then, the positive equilibrium point $E\left(y_{1}^{*}, y_{2}^{*}\right)$ of (7) is locally asymptotically stable for all $\tau \geq 0$.

Now, we consider the existence of Hopf bifurcation at the positive equilibrium of (7).

When $k=0$, we have

$$
r^{2}-q^{2}=\left(A_{1} B_{2}+A_{2} B_{1}\right)\left(A_{1} B_{2}-A_{2} B_{1}\right),
$$

where $A_{1} B_{2}-A_{2} B_{1}>0$.

Hence, if $A_{1} B_{2}+A_{2} B_{1}<0$, then $r^{2}-q^{2}<0$, and (23) has a unique positive root $\omega_{+}^{0}$, where

$$
\omega_{+}^{0}=\left\{\frac{1}{2}\left[2 r-p^{2}+\sqrt{\left(p^{2}-2 r\right)^{2}-4\left(r^{2}-p^{2}\right)}\right]\right\}^{1 / 2},
$$

and $z_{0}=\left(\omega_{+}^{0}\right)^{2}$. Equation (18) with $k=0$ has a pair of purely imaginary roots $\pm i \omega_{+}^{0}$. By (22), we can get the values for $\tau$ under this scenario:

$$
\tau_{j}^{0}=\frac{1}{\omega_{+}^{0}} \arctan \left\{\frac{p \omega_{+}^{0}}{\left(\omega_{+}^{0}\right)^{2}-r}\right\}+\frac{j \pi}{\omega_{+}^{0}}, \quad j \in \mathbb{N}_{0} .
$$

Hence, all roots of (18), except $\pm i \omega_{+}^{0}$, have no zero real parts. According to Theorem 2.7 by Li et al. [2], we know that if (H1) and $A_{1} B_{2}+A_{2} B_{1}<0$ hold, then the equilibrium $E\left(y_{1}^{*}, y_{2}^{*}\right)$ remains stable for $\tau<\tau_{0}^{0}=\min \left\{\tau_{j}^{0}\right\}, j \in \mathbb{N}_{0}$, and we verify that

$$
\left.\frac{\mathrm{d}(\operatorname{Re} \lambda)}{\mathrm{d} \tau}\right|_{\tau=\tau_{0}^{0}}>0
$$

where $\lambda(\tau)=\alpha(\tau)+i \omega(\tau)$ is a root of $(18), \alpha\left(\tau_{j}^{0}\right)=0$, and $\omega\left(\tau_{j}^{0}\right)=\omega_{0}$.

Apparently, the transversality condition holds in this case, and there is a sign of Hopf bifurcation at $\omega=\omega_{0}$ and $\tau=\tau_{0}^{0}$.

Furthermore, we discuss the case when $k \geq 1$. Let $\lambda=$ $i \omega_{1}\left(\omega_{1}>0\right)$ be a root of (18) with $k \geq 1$. Similarly, we have

$$
\omega_{1}^{4}+\left(p^{2}-2 r\right) \omega_{1}^{2}+r^{2}-q^{2}=0 .
$$

By performing some calculations, we can get

$$
r^{2}-q^{2} \geq\left(-A_{1}+D_{1}\right)^{2}\left(-B_{2}+D_{2}\right)^{2}-A_{2}^{2} B_{1}^{2} \text {. }
$$

It is easy to see that if the following condition holds,

$$
\text { (H3): }\left(-A_{1}+D_{1}\right)\left(-B_{2}+D_{2}\right)>A_{2}\left|B_{1}\right|,
$$

then (18) has no purely imaginary roots for $k \geq 1$. Thus, the following conclusions are true. 
Theorem 2. Assume that condition (H1) holds. $\omega_{+}^{0}$ and $\tau_{j}^{0}\left(j \in \mathbb{N}_{0}\right)$ be given by (29) and (30), respectively. Then, for system (7), the following conclusions are valid:

(i) When (H2) holds, the positive equilibrium $E$ is locally asymptotically stable for all $\tau \geq 0$

(ii) When $A_{1} B_{2}+A_{2} B_{1}<0$ and (H3) holds, the positive equilibrium $E$ is locally asymptotically stable if $\tau \in\left[0, \tau_{0}^{0}\right)$ and unstable if $\tau>\tau_{0}^{0}$

(iii) When $\tau=\tau_{j}^{0}\left(j \in \mathbb{N}_{0}\right)$, (7) occurs as Hopf bifurcation at $E$

By the same measure, we now make the following assumptions for a certain $k_{0} \in \mathbb{N}_{0} \backslash\{0\}$ :

$$
\begin{aligned}
& (H 4)\left(-A_{1}+D_{1} k_{0}^{2}\right)\left(-B_{2}+D_{2} k_{0}^{2}\right)+A_{2} B_{1}<0, \\
& (H 5)\left(-A_{1}+D_{1}\left(k_{0}-1\right)^{2}\right)\left(-B_{2}+D_{2}\left(k_{0}-1\right)^{2}\right)>\left|A_{2} B_{1}\right|, \\
& (H 6)\left(-A_{1}+D_{1}\left(k_{0}+1\right)^{2}\right)\left(-B_{2}+D_{2}\left(k_{0}+1\right)^{2}\right)>\left|A_{2} B_{1}\right| .
\end{aligned}
$$

Under condition (H4), equation (23) with $k=k_{0}$ has only a positive solution:

$$
\omega_{+}^{k_{0}}=\frac{\sqrt{2}}{2}\left[2 r-p^{2}+\sqrt{\left(2 r-p^{2}\right)^{2}-4\left(r^{2}-q^{2}\right)}\right]_{k=k_{0}}^{1 / 2} .
$$

Hence, when (H5) and (H6) hold, for $k \in\{1,2, \ldots$, $\left.k_{0}-1\right\}$, we can deduce that

$$
\left(-A_{1}+D_{1} k^{2}\right)\left(-B_{2}+D_{2} k^{2}\right)+A_{2} B_{1} \geq\left(-A_{1}+D_{1}\left(k_{0}-1\right)^{2}\right)\left(-B_{2}+D_{2}\left(k_{0}-1\right)^{2}\right)-\left|A_{2} B_{1}\right|>0
$$

and for $k \in\left\{k_{0}+1, k_{0}+2, \cdots\right\}$,

$$
\left(-A_{1}+D_{1} k^{2}\right)\left(-B_{2}+D_{2} k^{2}\right)+A_{2} B_{1} \geq\left(-A_{1}+D_{1}\left(k_{0}+1\right)^{2}\right)\left(-B_{2}+D_{2}\left(k_{0}+1\right)^{2}\right)-\left|A_{2} B_{1}\right|>0 .
$$

Therefore, it is easy to show that (18) with $k \in \mathbb{N}_{0} \backslash\left\{k_{0}\right\}$ has no purely imaginary roots, and it follows from (22) that

$$
\tau_{j}^{k_{0}}=\frac{1}{\omega_{+}^{k_{0}}} \arctan \left\{\frac{p w_{+}^{k_{0}}}{\left(\omega_{+}^{k_{0}}\right)^{2}-r}\right\}+\frac{j \pi}{\omega_{+}^{k_{0}}}, \quad j \in \mathbb{N}_{0} .
$$

Lemma 1. Assume that conditions (H1) and (H4)-(H6) hold; then, (18) with $k=k_{0}$ has a pair of purely imaginary roots $\pm i \omega_{+}^{k_{0}}$ with $\tau=\tau_{j}^{k_{0}}\left(j \in \mathbb{N}_{0}\right)$, and all roots of (18), except $\pm i \omega_{+}^{k_{0}}$, have no zero real parts.

Let $\lambda(\tau)=\alpha(\tau)+i \omega(\tau)$ be a root of (18) near $\tau=\tau_{j}^{k_{0}}$ satisfying $\alpha\left(\tau_{j}^{k_{0}}\right)=0$ and $\omega\left(\tau_{j}^{k_{0}}\right)=\omega_{+}^{k_{0}}$ for $j \in \mathbb{N}_{0}$; then, the following transversality condition is obtained.
Lemma 2. Assume that conditions (H1) and (H4)-(H6) hold. Then,

$$
\operatorname{sign}\left\{\operatorname{Re}\left(\frac{\mathrm{d} \lambda}{\mathrm{d} \tau}\right)\right\}_{\tau=\tau_{j}^{k_{0}}}>0
$$

Proof. Differentiating both sides of (18) with respect to $\tau$ yields

$$
\left(\frac{\mathrm{d} \lambda}{\mathrm{d} \tau}\right)^{-1}=\frac{\left\{2 \lambda+\left[D_{1} k_{0}^{2}+D_{2} k_{0}^{2}-\left(A_{1}+B_{2}\right)\right]\right\} e^{\lambda \tau}}{-\lambda A_{2} B_{1}}-\frac{\tau}{\lambda}
$$

It follows that

$$
\begin{aligned}
\operatorname{Re}\left(\frac{\mathrm{d} \lambda}{\mathrm{d} \tau}\right)_{\tau=\tau_{j}^{k_{0}}}^{-1} & =\operatorname{Re}\left[\frac{\left\{2 \lambda+\left[D_{1} k_{0}^{2}+D_{2} k_{0}^{2}-\left(A_{1}+B_{2}\right)\right]\right\} e^{\lambda \tau}}{-\lambda A_{2} B_{1}}-\frac{\tau}{\lambda}\right]_{\tau=\tau_{j}^{k_{0}}} \\
& =\operatorname{Re}\left\{\frac{\left[2 i \omega_{+}^{k_{0}}+\left(D_{1} k_{0}^{2}+D_{2} k_{0}^{2}-\left(A_{1}+B_{2}\right)\right)\right] e^{i \tau_{j}^{k_{0}} \omega_{+}^{k_{0}}}}{-i \omega_{+}^{k_{0}} A_{2} B_{1}}\right\} \\
& =\operatorname{Re}\left\{\frac{\left[-2 \omega_{+}^{k_{0}}+i\left(D_{1} k_{0}^{2}+D_{2} k_{0}^{2}-\left(A_{1}+B_{2}\right)\right)\right] e^{i \tau_{j}^{k_{0}} \omega_{+}^{k_{0}}}}{\omega_{+}^{k_{0}} A_{2} B_{1}}\right\} \\
& =\frac{-2 \omega_{+}^{k_{0}} \cos \tau_{j}^{k_{0}} \omega_{+}^{k_{0}}-\left(D_{1} k_{0}^{2}+D_{2} k_{0}^{2}-\left(A_{1}+B_{2}\right)\right) \sin \tau_{j}^{k_{0}} \omega_{+}^{k_{0}}}{\omega_{+}^{k_{0}} A_{2} B_{1}} .
\end{aligned}
$$


According to the above analysis,

$$
\operatorname{sign}\left\{\operatorname{Re}\left(\frac{\mathrm{d} \lambda}{\mathrm{d} \tau}\right)\right\}_{\tau=\tau_{j}^{k_{0}}}=\operatorname{sign}\left\{\operatorname{Re}\left(\frac{\mathrm{d} \lambda}{\mathrm{d} \tau}\right)^{-1}\right\}_{\tau=\tau_{j}^{k_{0}}}>0 .
$$

From Lemmas 1 and 2, and the Hopf bifurcation theorem [28], we can have the following result.

Theorem 3. Assume that condition (H1) holds. $\omega_{+}^{k_{0}}$ and $\tau_{j}^{k_{0}}$ are given by (36) and (39), respectively. Then, for system (7), the following results are true:

(i) When $\tau \in\left[0, \tau_{0}^{k_{0}}\right)$, the positive equilibrium point $E$ is asymptotically stable

(ii) When $\tau>\tau_{0}^{k_{0}}$, the positive equilibrium point $E$ is unstable (iii) When $\tau=\tau_{j}^{k_{0}}\left(j \in \mathbb{N}_{0}\right)$, (7) occurs as Hopf bifurcation at $E$

\section{Direction of Hopf Bifurcation and Stability of Bifurcating Periodic Solution}

In the preceding section, we obtained a series of periodic solutions bifurcating from the positive constant steady state $E$ of (7) when $\tau$ increases past the critical values $\tau_{j}^{k}\left(k=0, k_{0} ; j \in \mathbb{N}_{0}\right)$. In the present section, by using the normal form method and the center manifold theorem presented in [28-30], we compute the directions, stability, and periods of the bifurcating periodic solution. For brevity, we denote $\tau_{j}^{k}$ by $\bar{\tau}$ for some fixed $j \in \mathbb{N}_{0}$.

Let $u_{i}(t, x)=u_{i}(\tau t, x)(i=1,2)$ and $\tau=\hat{\tau}+\mu$. Then, system (7) can be transformed into

$$
\left\{\begin{array}{l}
\frac{\partial u_{1}(t, x)}{\partial t}=(\bar{\tau}+\mu) D_{1} \Delta u_{1}(t, x)+(\bar{\tau}+\mu)\left(u_{1}(t, x)+y_{1}^{*}+c_{1}\right)\left[-a_{1} u_{1}(t, x)-b_{1} u_{2}^{2}(t-1, x)-2 b_{1} y_{2}^{*} u_{2}(t-1, x)\right] \\
\frac{\partial u_{2}(t, x)}{\partial t}=(\tilde{\tau}+\mu) D_{2} \Delta u_{2}(t, x)+(\bar{\tau}+\mu)\left(u_{2}(t, x)+y_{2}^{*}+c_{2}\right)\left[-a_{2} u_{2}(t, x)+b_{2} u_{1}^{2}(t, x)+2 b_{2} y_{1}^{*} u_{1}(t, x)\right]
\end{array}\right.
$$

Let $u_{i}(t)=u_{i}(t, \cdot)(i=1,2)$ and $U=\left(u_{1}, u_{2}\right)^{T}$. Then, in the phase space $\ell=C([-\tau, 0], X)$, system (44) can be rewritten as

$$
\frac{\mathrm{d} U(t)}{\mathrm{d} t}=\hat{\tau} D \Delta U(t)+L(\bar{\tau})\left(U_{t}\right)+F\left(U_{t}, \mu\right),
$$

where $D=\operatorname{diag}\left(D_{1}, D_{2}\right), L: \ell \longrightarrow X$, and $F: \ell \times R \longrightarrow X$ are given as

$$
\begin{aligned}
L(\mu)(\phi) & =\mu\left(\begin{array}{c}
A_{1} \phi_{1}(0)+B_{1} \phi_{2}(-1) \\
A_{2} \phi_{1}(0)+B_{2} \phi_{2}(0)
\end{array}\right), \\
F(\phi, \mu) & =\mu D \Delta \phi(0)+L(\mu)(\phi)+f(\phi, \mu),
\end{aligned}
$$

where

$$
\begin{aligned}
f(\phi, \mu) & =(\hat{\tau}+\mu)\left(\begin{array}{c}
f_{1}+f_{2} \\
f_{3}+f_{4}
\end{array}\right), \\
f_{1} & =-a_{1} \phi_{1}^{2}(0)-b_{1}\left(y_{1}^{*}+c_{1}\right) \phi_{2}^{2}(-1), \\
f_{2} & =-2 b_{1} y_{2}^{*} \phi_{1}(0) \phi_{2}(-1)-b_{1} \phi_{1}(0) \phi_{2}^{2}(-1), \\
f_{3} & =F_{3}, f_{4}=F_{4},
\end{aligned}
$$

for $\phi(\theta)=\left(\phi_{1}(\theta), \phi_{2}(\theta)\right)^{T} \in \ell$. From the discussion in the last section, we know that $(44)$ has an equilibrium $(0,0)$ and $\Lambda_{0}=\left\{-i \omega_{+}^{k} \hat{\tau}, i \omega_{+}^{k} \hat{\tau}\right\}\left(k=0, k_{0}\right)$ are a pair purely imaginary eigenvalues of characteristic equation of the linear equation

$$
\dot{U}(t)=\hat{\tau} D \Delta U(t)+L(\widehat{\tau})\left(U_{t}\right),
$$

and the functional differential equation

$$
\dot{z}(t)=L_{k}(\hat{\tau})\left(z_{t}\right)
$$

where $L_{k}(\hat{\tau})$ is a continuous linear operator mapping $C\left([-1,0], \mathbb{R}^{2}\right)$ into $\mathbb{R}^{2}$.

According to the Riesz representation theorem, for the ordinary functional differential equation,

$$
\dot{X}(t)=-\tilde{\tau} D k^{2} X(t)+L(\widehat{\tau})\left(X_{t}\right)
$$

there exists a $2 \times 2$ matrix function of the bounded variation $\eta^{(k)}(\theta, \widehat{\tau}), \theta \in[-1,0]$, such that

$$
-\tilde{\tau} D k^{2} \phi(0)+L(\widehat{\tau})(\phi)=\int_{-1}^{0} d\left[\eta^{(k)}(\theta, \bar{\tau})\right] \phi(\theta),
$$

where $\phi(\theta) \in C\left([-1,0], \mathbb{R}^{2}\right)$. In fact, we can take

$$
\eta^{(k)}(\theta, \hat{\tau})=\left\{\begin{array}{cc}
\hat{\tau}\left(\begin{array}{cc}
-D_{1} k^{2}+A_{1} & 0 \\
A_{2} & -D_{2} k^{2}+B_{2}
\end{array}\right), & \theta=0, \\
0, & \theta \in(-1,0), \\
-\hat{\tau}\left(\begin{array}{cc}
0 & B_{1} \\
0 & 0
\end{array}\right), & \theta=-1 .
\end{array}\right.
$$

Let $A(\widehat{\tau})$ be the infinitesimal generator corresponding to (50) and $A^{*}$ be the formal adjoint of $A(\widehat{\tau})$ under the bilinear inner product 


$$
\begin{aligned}
\langle\psi(s), \phi(\theta)\rangle & =\psi(0) \phi(0)-\int_{-1}^{0} \int_{\xi=0}^{\theta} \psi(\xi-\theta) d\left[\eta^{(k)}(\theta, \widehat{\tau})\right] \phi(\xi) \mathrm{d} \xi \\
& =\psi(0) \phi(0)+\bar{\tau} \int_{-1}^{0} \psi(\xi+1)\left(\begin{array}{cc}
0 & B_{1} \\
0 & 0
\end{array}\right) \phi(\xi) \mathrm{d} \xi
\end{aligned}
$$

for $\phi \in C\left([-1,0], \mathbb{R}^{2}\right)$, and $\psi \in C\left([0,1], \mathbb{R}^{2}\right)$. Then, $A(\bar{\tau})$ and $A^{*}$ are a pair of adjoint operators [31]. Since $\pm i \omega_{+}^{k} \bar{\tau}$ are a pair of purely imaginary eigenvalues of $A(\bar{\tau})$, they are also a pair of eigenvalues of $A^{*}$. Let $P$ and $P^{*}$ be the center spaces of $A(\bar{\tau})$ and $A^{*}$, respectively, associated with $\Lambda_{0}$. Thus, $P^{*}$ is the adjoint space of $P$ and $\operatorname{dim} P=\operatorname{dim} P^{*}=2$ [30].

Lemma 3. Let

$$
\begin{gathered}
\xi=\frac{i \omega_{+}^{k}+D_{1} k^{2}-A_{1}}{B_{1} e^{-i \omega_{+}^{k} \bar{\tau}},} \\
\eta=\frac{-i \omega_{+}^{k}+D_{1} k^{2}+A_{1}}{A_{2}} .
\end{gathered}
$$

Then, $p_{1}(\theta)=e^{i \omega_{+}^{k} \hat{\tau} \theta}(1, \xi)^{T}, p_{2}(\theta)=\overline{p_{1}(\theta)}, \theta \in[-1,0]$ is a basis of $P$ associated with $\Lambda_{0}$, and $q_{1}(s)=$ $(1, \eta) e^{-i \omega_{+}^{k} \bar{\tau} s}, q_{2}(s)=\overline{q_{1}(s)}, s \in[0,1]$ is a basis of $Q$ associated with $\Lambda_{0}$.

Let $\Phi=\left(\Phi_{1}, \Phi_{2}\right)$ and $\Psi^{*}=\left(\Psi_{1}^{*}, \Psi_{2}^{*}\right)^{T}$ with $\Phi_{1}(\theta)=$ $\left(p_{1}(\theta)+p_{2}(\theta)\right) / 2, \Phi_{2}(\theta)=\left(p_{1}(\theta)-p_{2}(\theta)\right) / 2 i$, and $\Psi_{1}^{*}(s)$ $=\left(q_{1}(s)+q_{2}(s)\right) / 2, \Psi_{2}^{*}(s)=\left(q_{1}(s)-q_{2}(s)\right) / 2 i$.

Through calculation, we can get the following concrete expressions:

$$
\begin{aligned}
& \Phi_{1}(\theta)=\left(\begin{array}{c}
\operatorname{Re}\left\{e^{i \omega_{+}^{k} \bar{\tau} \theta}\right\} \\
\operatorname{Re}\left\{\xi e^{i \omega_{+}^{k} \bar{\tau} \theta}\right\}
\end{array}\right)=\left(\begin{array}{c}
\cos \omega_{+}^{k} \bar{\tau} \theta \\
\frac{M_{1} \cos \omega_{+}^{k} \bar{\tau} \theta-M_{2} \sin \omega_{+}^{k} \bar{\tau} \theta}{B_{1}}
\end{array}\right), \\
& \Phi_{2}(\theta)=\left(\begin{array}{c}
\operatorname{Im}\left\{e^{i \omega_{+}^{k} \bar{\tau} \theta}\right\} \\
\operatorname{Im}\left\{\xi e^{i \omega_{+}^{k} \hat{\tau}}\right\}
\end{array}\right)=\left(\begin{array}{c}
\sin \omega_{+}^{k} \omega_{+}^{k} \bar{\tau} \theta \\
\frac{M_{1} \sin \omega_{+}^{k} \bar{\tau} \theta+M_{2} \cos \omega_{+}^{k} \bar{\tau} \theta}{B_{1}}
\end{array}\right), \\
& \Psi_{1}^{*}(s)=\left(\begin{array}{c}
\operatorname{Re}\left\{e^{-i \omega_{+}^{k} \hat{\tau} s}\right\} \\
\operatorname{Re}\left\{\eta e^{-i \omega_{+}^{k} \hat{\tau} s}\right\}
\end{array}\right)^{T}=\left(\begin{array}{c}
\cos \omega_{+}^{k} \hat{\tau} s \\
-\frac{M_{3}}{A_{2}}
\end{array}\right)^{T}, \\
& \Psi_{2}^{*}(s)=\left(\begin{array}{c}
\operatorname{Im}\left\{e^{-i \omega_{+}^{k} \bar{\tau} s}\right\} \\
\operatorname{Im}\left\{\eta e^{-i \omega_{+}^{k} \hat{\tau} s}\right\}
\end{array}\right)^{T}=\left(\begin{array}{c}
-\sin \omega_{+}^{k} \bar{\tau} s \\
-\frac{M_{4}}{A_{2}}
\end{array}\right)^{T},
\end{aligned}
$$

for $\theta \in[-1,0]$ and $s \in[0,1]$. Also, 


$$
\begin{aligned}
& M_{1}=\left(D_{1} k^{2}-A_{1}\right) \cos \omega_{+}^{k} \bar{\tau}-\omega_{+}^{k} \sin \omega_{+}^{k} \bar{\tau}, \\
& M_{2}=\omega_{+}^{k} \cos \omega_{+}^{k} \hat{\tau}+\left(D_{1} k^{2}-A_{1}\right) \sin \omega_{+}^{k} \bar{\tau}, \\
& M_{3}=\left(D_{1} k^{2}+A_{1}\right) \cos \omega_{+}^{k} \bar{\tau} s+\omega_{+}^{k} \sin \omega_{+}^{k} \bar{\tau} s, \\
& M_{4}=-\left(D_{1} k^{2}+A_{1}\right) \sin \omega_{+}^{k} \bar{\tau} s+\omega_{+}^{k} \cos \omega_{+}^{k} \bar{\tau} s .
\end{aligned}
$$

Then, by (53) and some calculations, we can further obtain

$$
\begin{aligned}
& \left\langle\Psi_{1}^{*}, \Phi_{1}\right\rangle=1-\frac{\left(D_{1} k^{2}+A_{1}\right) M_{1}}{A_{2} B_{1}}+\bar{\tau}\left[\left(\frac{\sin \omega_{+}^{k} \hat{\tau}}{2 \omega_{+}^{k} \bar{\tau}}+\frac{\cos \omega_{+}^{k} \hat{\tau}}{2}\right) M_{1}+\frac{\sin \omega_{+}^{k} \hat{\tau}}{2} M_{2}\right], \\
& \left\langle\Psi_{1}^{*}, \Phi_{2}\right\rangle=-\frac{\left(D_{1} k^{2}+A_{1}\right) M_{2}}{A_{2}}+\bar{\tau}\left[\left(\frac{\sin \omega_{+}^{k} \hat{\tau}}{2 \omega_{+}^{k} \bar{\tau}}+\frac{\cos \omega_{+}^{k} \hat{\tau}}{2}\right) M_{2}-\frac{\sin \omega_{+}^{k} \hat{\tau}}{2} M_{1}\right], \\
& \left\langle\Psi_{2}^{*}, \Phi_{1}\right\rangle=-\frac{\omega_{+}^{k} M_{1}}{A_{2} B_{1}}-\widehat{\tau}\left[-\left(\frac{\cos \omega_{+}^{k} \bar{\tau}}{2}-\frac{\sin \omega_{+}^{k} \hat{\tau}}{2 \omega_{+}^{k} \bar{\tau}}\right) M_{2}+\frac{\sin \omega_{+}^{k}}{2} M_{1}\right], \\
& \left\langle\Psi_{2}^{*}, \Phi_{2}\right\rangle=-\frac{\omega_{+}^{k} M_{2}}{A_{2} B_{1}}-\widehat{\tau}\left[\left(\frac{\cos \omega_{+}^{k} \tau}{2}-\frac{\sin \omega_{+}^{k} \tau}{2 \omega_{+}^{k} \tau}\right) M_{1}+\frac{\sin \omega_{+}^{k} \bar{\tau}}{2} M_{2}\right] .
\end{aligned}
$$

Then, we define

$$
\left(\Psi^{*}, \Phi\right)=\left(\Psi_{j}^{*}, \Phi_{k}\right)=\left(\begin{array}{ll}
\left(\Psi_{1}^{*}, \Phi_{1}\right) & \left(\Psi_{1}^{*}, \Phi_{2}\right) \\
\left(\Psi_{2}^{*}, \Phi_{1}\right) & \left(\Psi_{2}^{*}, \Phi_{2}\right)
\end{array}\right),
$$

and structure a new basis $\Psi$ for $Q$ as

$$
\Psi=\left(\Psi_{1}, \Psi_{2}\right)^{T}=\left(\Psi^{*}, \Phi\right)^{-1} \Psi^{*} .
$$

Obviously, $(\Psi, \Phi)=I_{2}$ is a second-order unit matrix. Furthermore, we define $f_{k}=\left(\beta_{k}^{1}, \beta_{k}^{2}\right)$ and $c \cdot f_{k}=c_{1} \beta_{k}^{1}+$ $c_{2} \beta_{k}^{2}$, where $\beta_{k}^{1}=\left(\begin{array}{c}\cos k x \\ 0\end{array}\right), \beta_{k}^{2}=\left(\begin{array}{c}0 \\ \cos k x\end{array}\right)$, and $c=\left(c_{1}\right.$, $\left.c_{2}\right)^{T} \in C([-1,0], X)$. Thus, the center subspace of linear equation (48) is given as

$$
P_{C N} \ell(\phi)=\Phi\left(\Psi,\left\langle\phi, f_{k}\right\rangle\right) \cdot f_{k}, \phi \in \ell,
$$

where $\ell=P_{C N} \ell \oplus P_{s} \ell$ and $P_{s} \ell$ is the complementary subspace of $P_{C N} \ell$ in $\ell$.

Let $A_{\tilde{\tau}}$ be the infinitesimal generator corresponding to (48); then, (44) can be rewritten in the following form:

$$
\dot{U}_{t}=A_{\bar{\tau}} U_{t}+R\left(\mu, U_{t}\right) \text {, }
$$

where

$$
R\left(\mu, U_{t}\right)= \begin{cases}0, & \theta \in[-1,0], \\ F\left(U_{t}, \mu\right), & \theta=0 .\end{cases}
$$

Using the decomposition $\ell=P_{C N} \ell \oplus P_{s} \ell$ and (60), the solution of (45) on the center manifold can be rewritten as

$$
U_{t}=\Phi\left(\begin{array}{l}
x_{1}(t) \\
x_{2}(t)
\end{array}\right) \cdot f_{k}+h\left(x_{1}, x_{2}, \mu\right),
$$

where $\quad\left(\begin{array}{l}x_{1}(t) \\ x_{2}(t)\end{array}\right)=\left(\Psi,\left\langle U_{t}, f_{k}\right\rangle\right), h\left(x_{1}, x_{2}, \mu\right) \in P_{s} \ell, \quad h$ $(0,0,0)=0$, and $D h(0,0,0)=0$. In fact, on the center manifold, the solution of (45) is given by

$$
U_{t}=\Phi\left(\begin{array}{l}
x_{1}(t) \\
x_{2}(t)
\end{array}\right) \cdot f_{k}+h\left(x_{1}, x_{2}, 0\right) .
$$

Let $z=x_{1}-i x_{2}, \Psi(0)=\left(\Psi_{1}(0), \Psi_{2}(0)\right)^{T}$. By the definitions of $\Phi_{1}$ and $\Phi_{2}$, it is easy to obtain $p_{1}=\Phi_{1}+i \Phi_{2}$, and we further obtain

$$
\Phi\left(\begin{array}{c}
x_{1}(t) \\
x_{2}(t)
\end{array}\right) \cdot f_{k}=\left(\Phi_{1}, \Phi_{2}\right)\left(\begin{array}{c}
\frac{1}{2}(z+\bar{z}) \\
\frac{i}{2}(z-\bar{z})
\end{array}\right) \cdot f_{k}=\frac{1}{2}\left(p_{1} z+\overline{p_{1}} \bar{z}\right) \cdot f_{k}
$$


Therefore, (64) can be expressed as

$$
U_{t}=\frac{1}{2}\left(p_{1} z+\overline{p_{1}} \bar{z}\right) \cdot f_{k}+W(z, \bar{z}),
$$

where $W(z, \bar{z})=h((z+\bar{z}) / 2, i(z-\bar{z}) / 2,0)$. By [30], we can deduce that $z$ satisfies

$$
\dot{z}=i \omega_{+}^{k} \hat{\tau} z+g(z, \bar{z}),
$$

where

$$
g(z, \bar{z})=\left(\Psi_{1}(0)-i \Psi_{2}(0)\right)\left\langle f\left(U_{t}, 0\right), f_{k}\right\rangle .
$$

Let

$$
\begin{aligned}
& W(z, \bar{z})=W_{20} \frac{z^{2}}{2}+W_{11} z \bar{z}+W_{02} \frac{\bar{z}^{2}}{2}+\cdots \\
& g(z, \bar{z})=g_{20} \frac{z^{2}}{2}+g_{11} z \bar{z}+g_{02} \frac{\bar{z}^{2}}{2}+g_{21} \frac{z^{2} \bar{z}}{2}+\cdots
\end{aligned}
$$

From (47) and (64), (66) can be rewritten as

$$
\begin{aligned}
U_{t} & =\frac{1}{2}\left[z e^{i w_{+}^{k} \bar{\tau} \theta}\left(\begin{array}{c}
1 \\
\xi
\end{array}\right)+\bar{z} e^{-i w_{+}^{k} \bar{\tau} \theta}\left(\begin{array}{c}
1 \\
\xi
\end{array}\right)\right] \cdot f_{k}+W(z, \bar{z}) \\
& =\frac{1}{2}\left(\begin{array}{c}
\left(z e^{i w_{+}^{k} \bar{\tau} \theta}+\bar{z} e^{-i w_{+}^{k} \bar{\tau} \theta}\right) \cos k x \\
\left(z \xi e^{i w_{+}^{k} \bar{\tau} \theta}+\bar{\xi} \bar{z} e^{-i w_{+}^{k} \bar{\tau} \theta}\right) \cos k x
\end{array}\right)+\left(\begin{array}{c}
W^{(1)}(z, \bar{z}) \\
W^{(2)}(z, \bar{z})
\end{array}\right) .
\end{aligned}
$$

Then,

$$
\begin{aligned}
& u_{1 t}(0)=\frac{1}{2}(z+\bar{z}) \cos k x+W_{20}^{(1)}(0) \frac{z^{2}}{2}+W_{11}^{(1)}(0) z \bar{z}+W_{02}^{(1)}(0) \frac{\bar{z}^{2}}{2}+\cdots ; \\
& u_{1 t}(-1)=\frac{1}{2}\left(z e^{-i \omega_{+}^{k} \bar{\tau}}+\bar{z} e^{i \omega_{+}^{k} \bar{\tau}}\right) \cos k x+W_{20}^{(1)}(-1) \frac{z^{2}}{2}+W_{11}^{(1)}(-1) z \bar{z}+W_{02}^{(1)}(-1) \frac{\bar{z}^{2}}{2}+\cdots ; \\
& u_{2 t}(0)=\frac{1}{2}(z \xi+\bar{z} \bar{\xi}) \cos k x+W_{20}^{(2)}(0) \frac{z^{2}}{2}+W_{11}^{(2)}(0) z \bar{z}+W_{02}^{(2)}(0) \frac{\bar{z}^{2}}{2}+\cdots ; \\
& u_{2 t}(-1)=\frac{1}{2}\left(z \xi e^{-i \omega_{+}^{k} \bar{\tau}}+\bar{z} \bar{\xi} e^{i \omega_{+}^{k} \bar{\tau}}\right) \cos k x+W_{20}^{(2)}(-1) \frac{z^{2}}{2}+W_{11}^{(2)}(-1) z \bar{z}+W_{02}^{(2)}(-1) \frac{\bar{z}^{2}}{2}+\cdots
\end{aligned}
$$

Therefore, we can have $f\left(U_{t}, 0\right)=\tau\left(\begin{array}{l}f_{11} \\ f_{22}\end{array}\right)$, where

$$
\begin{aligned}
f_{11}= & -a_{1} u_{1 t}^{2}(0)-b_{1}\left(y_{1}^{*}+c_{1}\right) u_{2 t}^{2}(-1)-2 b_{1} y_{2}^{*} u_{1 t}(0) u_{2 t}(-1)-b_{1} u_{1 t}(0) u_{2 t}^{2}(-1), \\
f_{22}= & -a_{2} u_{2 t}^{2}(0)+b_{2}\left(y_{2}^{*}+c_{2}\right) u_{1 t}^{2}(0)+2 b_{2} y_{1}^{*} u_{1 t}(0) u_{2 t}(0)+b_{2} u_{1 t}^{2}(0) u_{2 t}(0), \\
\left\langle f\left(U_{t}, 0\right), f_{k}\right\rangle= & \frac{\hat{\tau}}{2}\left(\begin{array}{c}
M_{11} \\
M_{12}
\end{array}\right) \frac{1}{\pi} \int_{0}^{\pi} \cos ^{3} k x d x \frac{z^{2}}{2}+\frac{\hat{\tau}}{4}\left(\begin{array}{c}
M_{21} \\
M_{22}
\end{array}\right) \frac{1}{\pi} \int_{0}^{\pi} \cos ^{3} k x d x z \bar{z} \\
& +\frac{\hat{\tau}}{2}\left(\begin{array}{c}
M_{31} \\
M_{32}
\end{array}\right) \frac{1}{\pi} \int_{0}^{\pi} \cos ^{3} k x d x \frac{\bar{z}^{2}}{2}+\hat{\tau}\left(\begin{array}{c}
M_{41} \\
M_{42}
\end{array}\right) \frac{z^{2} \bar{z}}{2}+\cdots,
\end{aligned}
$$

where 


$$
\begin{aligned}
& M_{11}=-a_{1}-b_{1}\left(y_{1}^{*}+c_{1}\right) \xi^{2} e^{-2 i \omega_{+}^{k} \bar{\tau}}-2 b_{1} y_{2}^{*} \xi e^{-i \omega_{+}^{k} \bar{\tau}}, \\
& M_{12}=-a_{2} \xi^{2}+b_{2}\left(y_{2}^{*}+c_{2}\right)+2 b_{2} y_{1}^{*} \xi \\
& M_{21}=-2 a_{1}-2 b_{1}\left(y_{1}^{*}+c_{1}\right) \xi \bar{\xi}-2 b_{1} y_{2}^{*}\left(\xi e^{-i \omega_{+}^{k} \bar{\tau}}+\bar{\xi} e^{i \omega_{+}^{k} \bar{\tau}}\right), \\
& M_{22}=-2 a_{2} \xi \bar{\xi}+2 b_{2}\left(y_{2}^{*}+c_{2}\right)+2 b_{2} y_{1}^{*}(\xi+\bar{\xi}), \\
& M_{31}=\overline{M_{11}}, M_{32}=\overline{M_{12}} \text {, } \\
& M_{41}=-a_{1}\left\langle\left(\frac{1}{2} W_{20}^{(1)}(0)+W_{11}^{(1)}(0)\right) \cos k x, \cos k x\right\rangle \\
& -b_{1}\left(y_{1}^{*}+c_{1}\right)\left\langle\left(\frac{\bar{\xi}}{2} W_{20}^{(2)}(-1) e^{i \omega_{+}^{k} \bar{\tau}}+\xi W_{11}^{(2)}(-1) e^{-i \omega_{+}^{k} \bar{\tau}}\right) \cos k x, \cos k x\right\rangle \\
& -2 b_{1} y_{2}^{*}\left\langle\left(\frac{1}{2} W_{11}^{(2)}(-1)+\frac{1}{4} W_{20}^{(2)}(-1)+\frac{\bar{\xi}}{4} W_{20}^{(1)}(0) e^{i \omega_{+}^{k} \bar{\tau}}+\frac{\xi}{4} W_{11}^{(1)}(0) e^{-i \omega_{+}^{k} \bar{\tau}}\right) \cos k x, \cos k x\right\rangle \\
& -b_{1}\left\langle\left(\xi \bar{\xi}+\frac{\xi^{2}}{2} e^{-2 i \omega_{+}^{k}} \bar{\tau}\right) \cos k x, \cos k x\right\rangle \\
& M_{42}=-a_{2}\left\langle\left(\xi W_{11}^{(2)}(0)+\frac{\bar{\xi}}{2} W_{20}^{(2)}(0)\right) \cos k x, \cos k x\right\rangle+b_{2}\left(y_{2}^{*}+c_{2}\right)\left\langle\left(W_{11}^{(2)}(0)+\frac{1}{2} W_{20}^{(1)}(0)\right) \cos k x, \cos k x\right\rangle \\
& +2 b_{2} y_{1}^{*}\left\langle\left(\frac{1}{2} W_{11}^{(2)}(0)+\frac{1}{4} W_{20}^{(2)}(0)+\frac{\bar{\xi}}{4} W_{20}^{(1)}(0)+\frac{\xi}{2} W_{11}^{(1)}(0)\right) \cos k x, \cos k x\right\rangle \\
& +b_{2}\langle(\bar{\xi}+2 \xi) \cos k x, \cos k x\rangle \text {, }
\end{aligned}
$$

$$
\left\langle W_{i j}^{(n)}(\theta), \cos k x\right\rangle=\frac{1}{\pi} \int_{0}^{\pi} W_{i j}^{(n)}(\theta)(x) \cos k x d x, \quad i+j=2, n=1,2
$$

Note that $\int_{0}^{\pi} \cos ^{3} k x \mathrm{~d} x=0$ for $\forall k \in \mathbb{N}$.

Let $\left(\psi_{1}, \psi_{2}\right)=\Psi_{1}(0)-i \Psi_{2}(0)$. Comparing the coefficient with that of $(70)$, we can obtain

$$
\begin{aligned}
& g_{20}= \begin{cases}0, & k \in \mathbb{N}, \\
\frac{\hat{\tau}}{2}\left(M_{11} \psi_{1}+M_{12} \psi_{2}\right), & k=0,\end{cases} \\
& g_{11}= \begin{cases}0, & k \in \mathbb{N}, \\
\frac{\hat{\tau}}{4}\left(M_{21} \psi_{1}+M_{22} \psi_{2}\right), & k=0,\end{cases} \\
& g_{02}=\frac{g_{20},}{g_{21}}=\hat{\tau} M_{41} \psi_{1}+\hat{\tau} M_{42} \psi_{2} .
\end{aligned}
$$

Next, we compute the terms $W_{20}(\theta)$ and $W_{11}(\theta)$ that appear in the equation for $g_{21}$. Using a method to the one used in [32, 33] and [27], it follows from (69) that

$$
\dot{W}(z, \bar{z})=W_{20} z \dot{z}+W_{11} \dot{z} \bar{z}+W_{11} z \dot{\bar{z}}+W_{02} \bar{z} \dot{z}+\cdots
$$

$$
A_{\bar{\tau}} W=A_{\bar{\tau}} W_{20} \frac{z^{2}}{2}+A_{\bar{\tau}} W_{11} z \bar{z}+A_{\bar{\tau}} W_{02} \frac{\bar{z}^{2}}{2}+\cdots
$$

In addition, we can get from [30] that

$$
\dot{W}=A_{\bar{\tau}} W+H(z, \bar{z}) \text {, }
$$

where

$$
\begin{aligned}
H(z, \bar{z})= & H_{20} \frac{z^{2}}{2}+H_{11} z \bar{z}+H_{02} \frac{\bar{z}^{2}}{2}+\cdots=X_{0} f\left(U_{t}, 0\right) \\
& -\Phi\left(\Psi,\left\langle X_{0} f\left(U_{t}, 0\right), f_{k}\right\rangle\right) \cdot f_{k} .
\end{aligned}
$$

Then, from (67) and (70)-(81), we have

$$
\left\{\begin{array}{l}
\left(2 i \omega_{+}^{k} \bar{\tau}-A_{\bar{\tau}}\right) W_{20}=H_{20}, \\
-A_{\bar{\tau}} W_{11}=H_{11}, \\
\left(-2 i \omega_{+}^{k} \bar{\tau}-A_{\bar{\tau}}\right) W_{02}=H_{02} .
\end{array}\right.
$$

Since $-A_{\bar{\tau}}$ has only two eigenvalues $\pm i \omega_{+}^{k} \hat{\tau}$, (82) has a unique solution $W_{i j}$ in $Q$ given by 


$$
\left\{\begin{array}{l}
W_{20}=\left(2 i \omega_{+}^{k} \bar{\tau}-A_{\bar{\tau}}\right)^{-1} H_{20}, \\
W_{11}=-A_{\bar{\tau}}^{-1} H_{11}, \\
W_{02}=\left(-2 i \omega_{+}^{k} \bar{\tau}-A_{\bar{\tau}}\right)^{-1} H_{02} .
\end{array}\right.
$$

$$
\begin{aligned}
H(z, \bar{z}) & =-\Phi(\theta) \Psi(0)\left\langle f\left(U_{t}, 0\right), f_{k}\right\rangle \cdot f_{k} \\
& =-\left(\frac{p_{1}(\theta)+p_{2}(\theta)}{2}, \frac{p_{1}(\theta)-p_{2}(\theta)}{2 i}\right)\left(\Psi_{1}(0), \Psi_{2}(0)\right)\left\langle f\left(U_{t}, 0\right), f_{k}\right\rangle \cdot f_{k} \\
& =-\frac{1}{2}\left[p_{1}(\theta)\left(\Psi_{1}(0)-i \Psi_{2}(0)\right)+p_{2}(\theta)\left(\Psi_{1}(0)+i \Psi_{2}(0)\right)\right]\left\langle f\left(U_{t}, 0\right), f_{k}\right\rangle \cdot f_{k} \\
& =-\frac{1}{2}\left(p_{1}(\theta) g_{20}+p_{2}(\theta) \overline{g_{02}}\right) \cdot f_{k} \frac{z^{2}}{2}-\frac{1}{2}\left(p_{1}(\theta) g_{11}+p_{2}(\theta) \overline{g_{11}}\right) \cdot f_{k} z \bar{z}+\cdots
\end{aligned}
$$

Hence, for $\theta \in[-1,0)$, we have

$$
\begin{aligned}
H_{20}(\theta)= \begin{cases}0, & k \in \mathbb{N}, \\
-\frac{1}{2}\left[p_{1}(\theta) g_{20}+p_{2}(\theta) \overline{g_{02}}\right] \cdot f_{0}, & k=0,\end{cases} \\
H_{11}(\theta)= \begin{cases}0, & k \in \mathbb{N}, \\
-\frac{1}{2}\left[p_{1}(\theta) g_{11}+p_{2}(\theta) \overline{g_{11}}\right] \cdot f_{0}, & k=0,\end{cases} \\
H(z, \bar{z})(0)=f\left(U_{t}, 0\right)-\Phi\left(\Psi,\left\langle X_{0} f\left(U_{t}, 0\right), f_{k}\right\rangle\right) \cdot f_{k}, \\
H_{20}(0)= \begin{cases}\frac{\hat{\tau}}{2}\left(\begin{array}{l}
M_{11} \\
M_{12}
\end{array}\right) \cos ^{2} k x, & k \in \mathbb{N}, \\
\frac{\hat{\tau}}{2}\left(\begin{array}{l}
M_{11} \\
M_{12}
\end{array}\right)-\frac{p_{1}(0) g_{20}+p_{2}(0) \overline{g_{02}}}{2} \cdot f_{0}, & k=0,\end{cases} \\
H_{11}(0)= \begin{cases}\bar{\tau}\left(\begin{array}{l}
M_{21} \\
4 \\
M_{22}
\end{array}\right) \cos ^{2} k x, & k \in \mathbb{N}, \\
\bar{\tau}\left(\begin{array}{l}
M_{21} \\
4
\end{array}\right)-\frac{p_{1}(0) g_{11}+p_{2}(0) \overline{g_{11}}}{2} \cdot f_{0}, & k=0 .\end{cases}
\end{aligned}
$$

From the definition of $A_{\bar{\tau}}$ and (82), we can get 


$$
\begin{array}{ll}
\dot{W}_{20}(\theta)=2 i \omega_{+}^{k} \hat{\tau} W_{20}(\theta)+\frac{1}{2}\left[p_{1}(\theta) g_{20}+p_{2}(\theta) \overline{g_{02}}\right] \cdot f_{k} . & (89)
\end{array} \quad E_{1}= \begin{cases}W_{20}(0), & k \in \mathbb{N}, \\
W_{20}(0)-\frac{1}{2}\left[\frac{i g_{20}}{\omega_{+}^{k} \bar{\tau}} p_{1}(0)+\frac{i \overline{g_{02}}}{3 \omega_{+}^{k} \bar{\tau}} p_{2}(0)\right] \cdot f_{0}, & k=0 .\end{cases}
$$

$W_{20}(\theta)=\frac{1}{2}\left[\frac{i g_{20}}{\omega_{+}^{k} \bar{\tau}} p_{1}(\theta)+\frac{i \overline{g_{02}}}{3 \omega_{+}^{k} \bar{\tau}} p_{2}(\theta)\right] \cdot f_{k}+E_{1} e^{2 i \omega_{+}^{k} \bar{\tau} \theta}$

where
By using the definition of $A_{\bar{\tau}}$ and combining (82) and (90), we can obtain the following equation:

$$
\begin{aligned}
& 2 i \omega_{+}^{k} \bar{\tau}\left[\frac{1}{2}\left(\frac{i g_{20}}{\omega_{+}^{0} \bar{\tau}} p_{1}(0) \cdot f_{0}+\frac{i \overline{g_{02}}}{3 \omega_{+}^{0} \bar{\tau}} p_{2}(0) \cdot f_{0}\right)+E_{1}\right]-\bar{\tau} D \Delta\left[\frac{1}{2}\left(\frac{i g_{20}}{\omega_{+}^{0} \bar{\tau}} p_{1}(0) \cdot f_{0}+\frac{i \overline{g_{02}}}{3 \omega_{+}^{0} \bar{\tau}} p_{2}(0) \cdot f_{0}\right)+E_{1}\right] \\
& -L(\bar{\tau})\left[\frac{1}{2}\left(\frac{i g_{20}}{\omega_{+}^{0} \bar{\tau}} p_{1}(\theta) \cdot f_{0}+\frac{i \overline{g_{02}}}{3 \omega_{+}^{0} \bar{\tau}} p_{2}(\theta) \cdot f_{0}\right)+E_{1} e^{2 i \omega_{+}^{0} \bar{\tau} \theta}\right] \\
= & \frac{\bar{\tau}}{2}\left(\begin{array}{l}
M_{11} \\
M_{12}
\end{array}\right)-\frac{1}{2}\left(p_{1}(0) g_{20}+p_{2}(0) \overline{g_{02}}\right) \cdot f_{0} .
\end{aligned}
$$

Note that

$$
\begin{aligned}
& \hat{\tau} D \Delta\left[p_{1}(0) \cdot f_{0}\right]+L(\bar{\tau})\left[p_{1}(\theta) \cdot f_{0}\right]=i \omega_{+}^{0} \bar{\tau} p_{1}(0) \cdot f_{0}, \\
& \bar{\tau} D \Delta\left[p_{2}(0) \cdot f_{0}\right]+L(\bar{\tau})\left[p_{2}(\theta) \cdot f_{0}\right]=i \omega_{+}^{0} \bar{\tau} p_{2}(0) \cdot f_{0} .
\end{aligned}
$$

Then, for $k \in \mathbb{N}_{0}$, we get

$2 i \omega_{+}^{k} \widehat{\tau} E_{1}-\widehat{\tau} D \Delta E_{1}-L(\widehat{\tau})\left(E_{1} e^{2 i \omega_{+}^{0} \hat{\tau} \theta}\right)=\frac{\hat{\tau}}{2}\left(\begin{array}{c}M_{11} \\ M_{12}\end{array}\right) \cos ^{2} k x$.

By (94), we have

$$
E_{1}=\frac{1}{2} E\left(\begin{array}{c}
M_{11} \\
M_{12}
\end{array}\right) \cos ^{2} k x,
$$

where

$E=\left(\begin{array}{cc}2 i \omega_{+}^{k}+a_{1}\left(y_{1}^{*}+c_{1}\right)+D_{1} k^{2} & -2 b_{1} y_{2}^{*}\left(y_{1}^{*}+c_{1}\right) e^{-2 i \omega_{+}^{k} \tau} \\ -2 b_{2} y_{1}^{*}\left(y_{2}^{*}+c_{2}\right) & 2 i \omega_{+}^{k}+a_{2}\left(y_{2}^{*}+c_{2}\right)+D_{2} k^{2}\end{array}\right)^{-1}$.

Similarly, we can also have

$$
\begin{aligned}
-\dot{W}_{11}(\theta) & =-\frac{1}{2}\left[p_{1}(\theta) g_{11}+p_{2}(\theta) \overline{g_{11}}\right] \cdot f_{k}, \quad \theta \in[-1,0), \\
W_{11}(\theta) & =\frac{1}{2}\left[\frac{-i g_{11}}{\omega_{+}^{k} \bar{\tau}} p_{1}(\theta)+\frac{i \overline{g_{11}}}{\omega_{+}^{k} \bar{\tau}} p_{2}(\theta)\right]+E_{2},
\end{aligned}
$$

where

$$
\begin{aligned}
& E_{2}=\frac{1}{4} E^{\prime}\left(\begin{array}{c}
M_{21} \\
M_{22}
\end{array}\right) \cos ^{2} k x, \\
& E^{\prime}=\left(\begin{array}{cc}
a_{1}\left(y_{1}^{*}+c_{1}\right)+D_{1} k^{2} & -2 b_{1} y_{2}^{*}\left(y_{1}^{*}+c_{1}\right) \\
-2 b_{2} y_{1}^{*}\left(y_{2}^{*}+c_{2}\right) & a_{2}\left(y_{2}^{*}+c_{2}\right)+D_{2} k^{2}
\end{array}\right)^{-1} .
\end{aligned}
$$

Substituting (90) and (98) into the expression of $g_{21}$, we can compute the following values:

$$
\begin{aligned}
c_{1}(0) & =\frac{i}{2 \omega_{+}^{k} \widehat{\tau}}\left(g_{20} g_{11}-2\left|g_{11}\right|^{2}-\frac{1}{3}\left|g_{02}\right|^{2}\right)+\frac{1}{2} g_{21}, \\
\mu_{2} & =-\frac{\operatorname{Re}\left\{c_{1}(0)\right\}}{\operatorname{Re}\left\{\lambda^{\prime}\left(\tau_{j}^{k}\right)\right\}}, \\
\beta_{2} & =2 \operatorname{Re}\left\{c_{1}(0)\right\}, \\
T_{2} & =-\frac{1}{\omega_{+}^{k} \tau}\left(\operatorname{Im}\left\{c_{1}(0)\right\}+\mu_{2} \operatorname{Im}\left\{\lambda^{\prime}\left(\tau_{j}^{k}\right)\right\}\right) .
\end{aligned}
$$

Through the above analysis, we can draw the following conclusions.

Theorem 3. Suppose conditions (H1)-(H6) hold. Then, the parameters $\mu_{2}, \beta_{2}$, and $T_{2}$ determine the properties of Hopf bifurcation, where $\mu_{2}$ determines the direction of the Hopf bifurcation, $\beta_{2}$ determines the stability of the bifurcating periodic solutions on the center manifold, and $T_{2}$ determines the period of the bifurcating periodic solutions, i.e., 


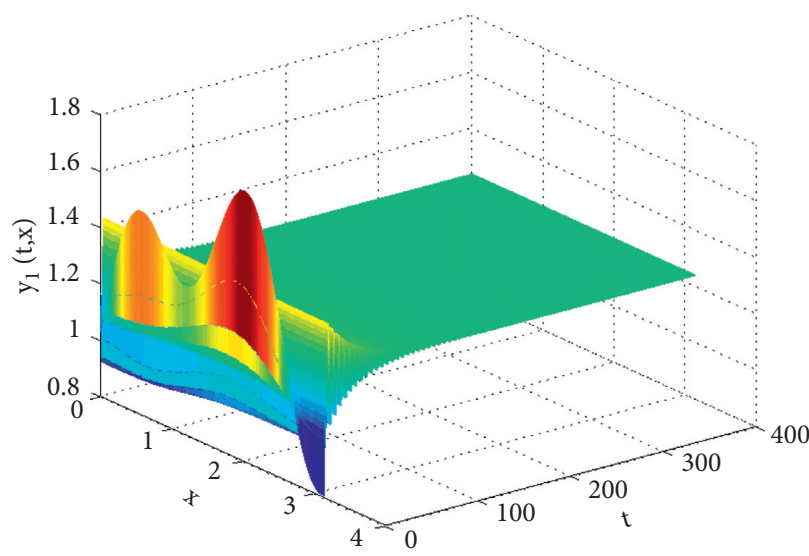

(a)

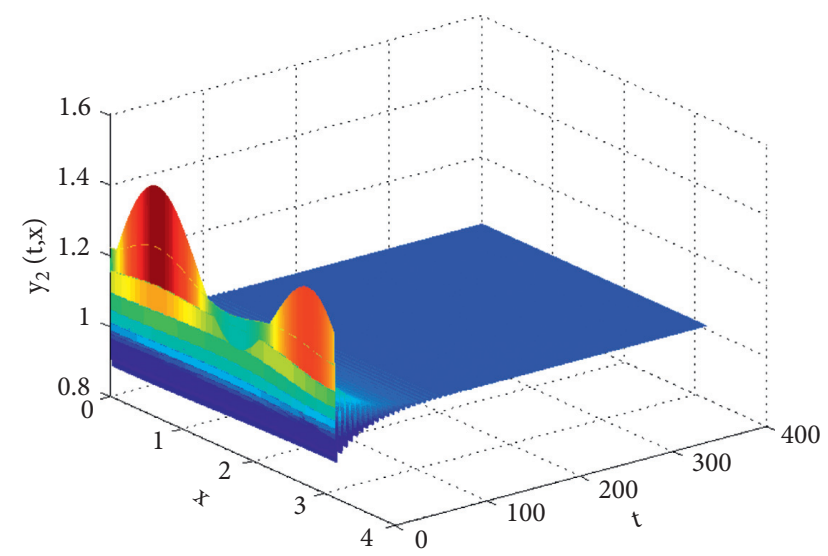

(b)

Figure 1: The positive constant steady state of the system with Neumann boundary is locally asymptotically stable for $\tau_{1}=0.9, \tau_{2}=0.9$, and $\tau=\tau_{1}+\tau_{2}<\tau_{0}^{0}=2.3633$. (a) Spatial distribution of $y_{1}(t, x)$. (b) Spatial distribution of $y_{2}(t, x)$.

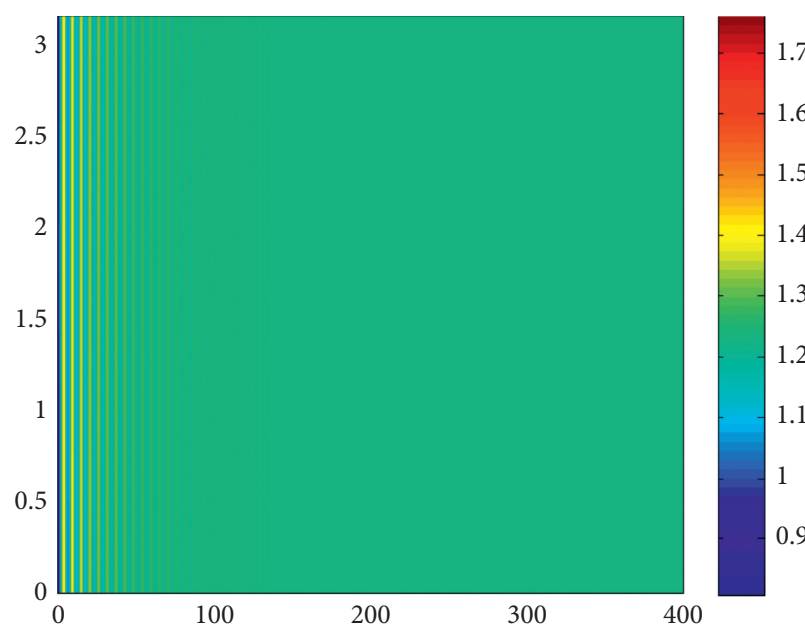

(a)

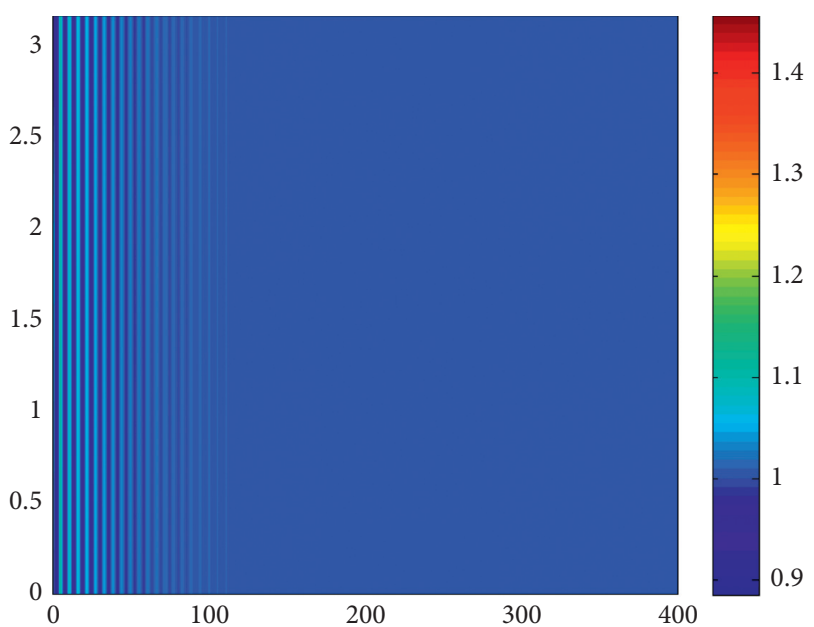

(b)

FiguRE 2: The spatial pattern of the system with Neumann boundary for $\tau_{1}=0.9, \tau_{2}=0.9$, and $\tau=\tau_{1}+\tau_{2}<\tau_{0}^{0}=2.3633$. (a) Spatial pattern of $y_{1}(t, x)$. (b) Spatial pattern of $y_{2}(t, x)$.

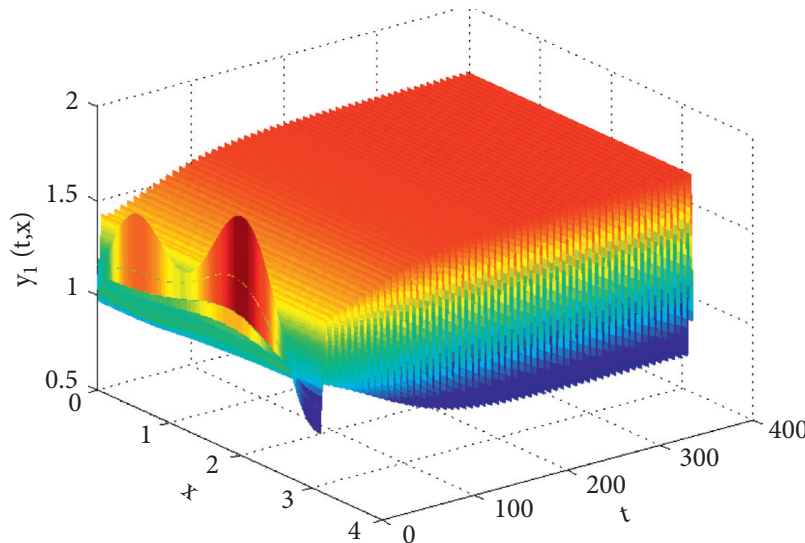

(a)

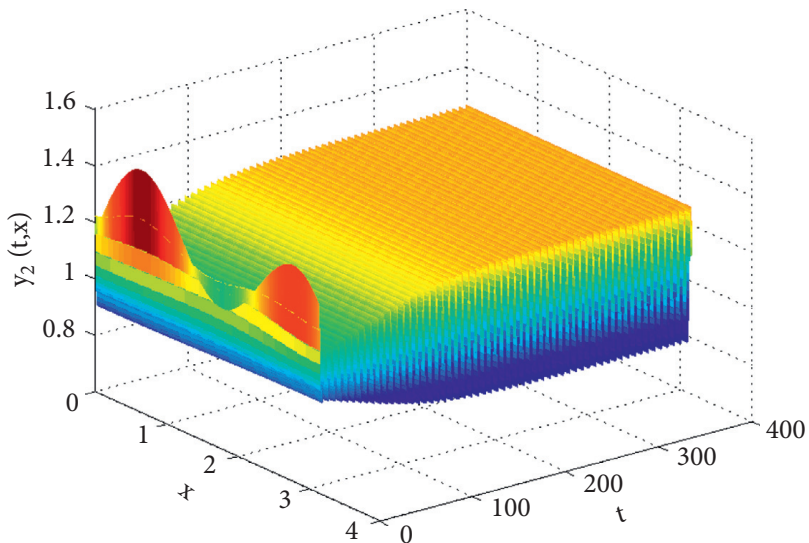

(b)

FIgURE 3: The positive constant steady state of the system with Neumann boundary loses its stability, and Hopf bifurcation occurs for $\tau_{1}=0.9, \tau_{2}=1.5$, and $\tau=\tau_{1}+\tau_{2}>\tau_{0}^{0}=2.3633$. (a) Spatial distribution of $y_{1}(t, x)$. (b) Spatial distribution of $y_{2}(t, x)$. 


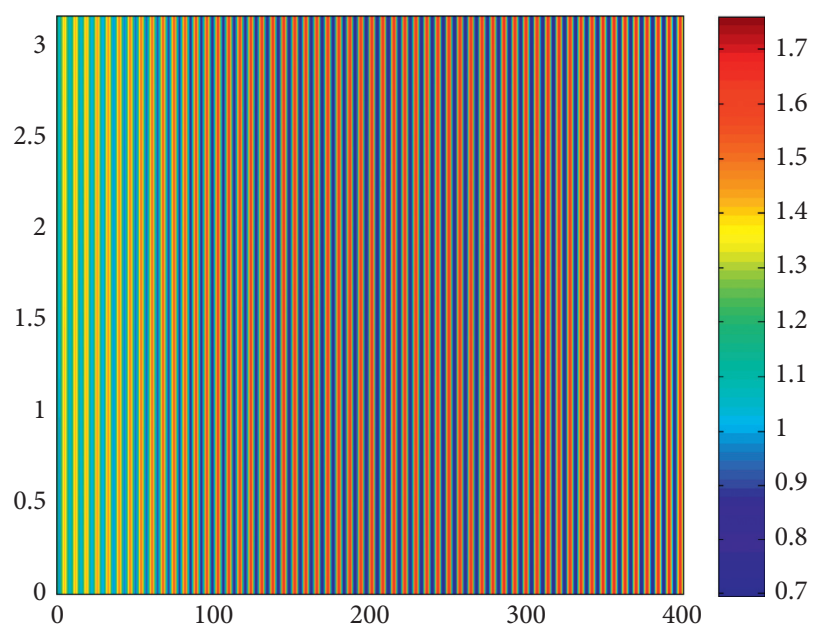

(a)

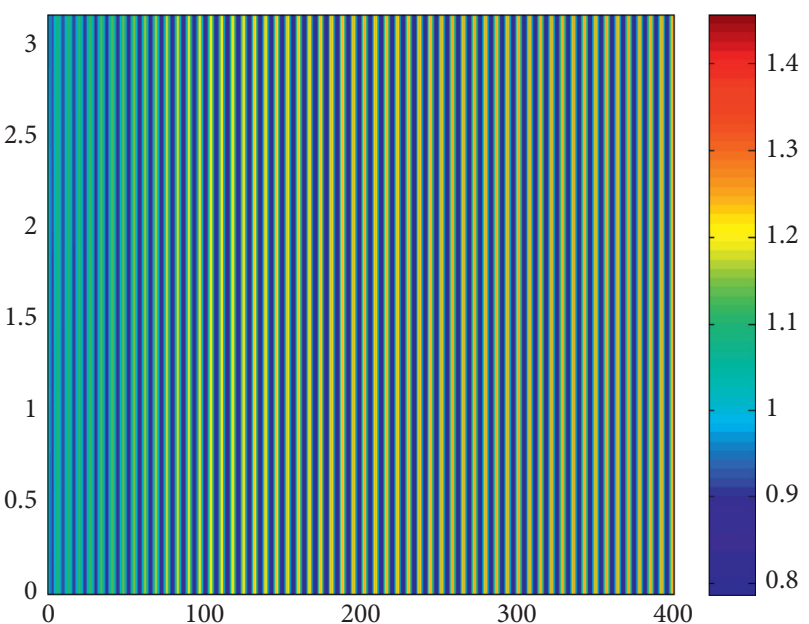

(b)

FIgURE 4: The spatial pattern of the system with Neumann boundary for $\tau_{1}=0.9, \tau_{2}=1.5$, and $\tau=\tau_{1}+\tau_{2}>\tau_{0}^{0}=2.3633$. (a) Spatial pattern of $y_{1}(t, x)$. (b) Spatial pattern of $y_{2}(t, x)$.

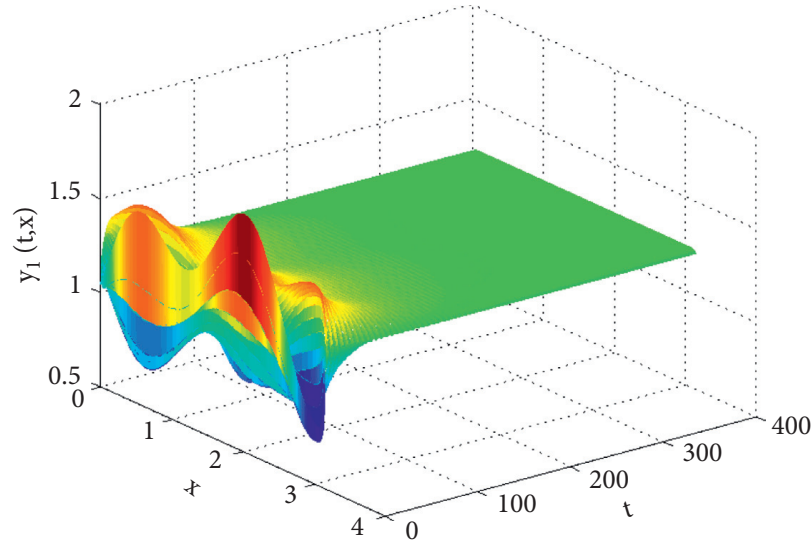

(a)

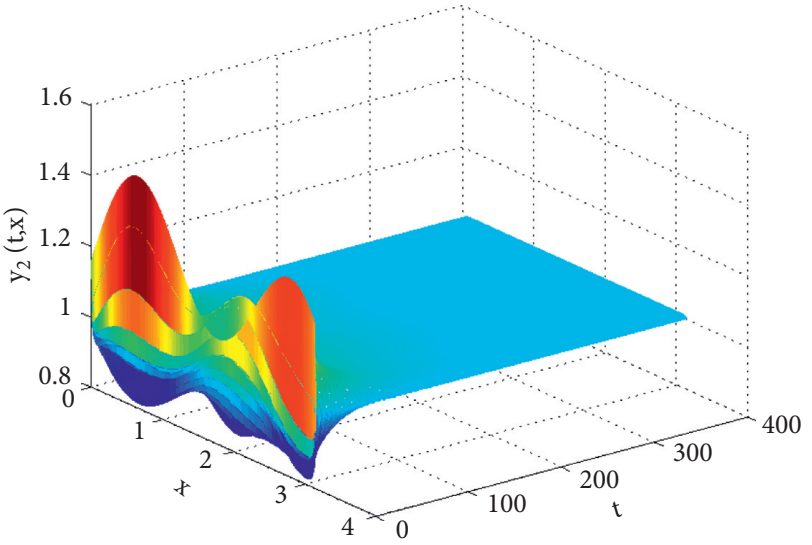

(b)

Figure 5: The positive constant steady state of the system with Dirichlet boundary is locally asymptotically stable for $\tau_{1}=0.9, \tau_{2}=0.9$, and $\tau=\tau_{1}+\tau_{2}<\tau_{0}^{0}=2.3633$. (a) Spatial distribution of $y_{1}(t, x)$. (b) Spatial distribution of $y_{2}(t, x)$.

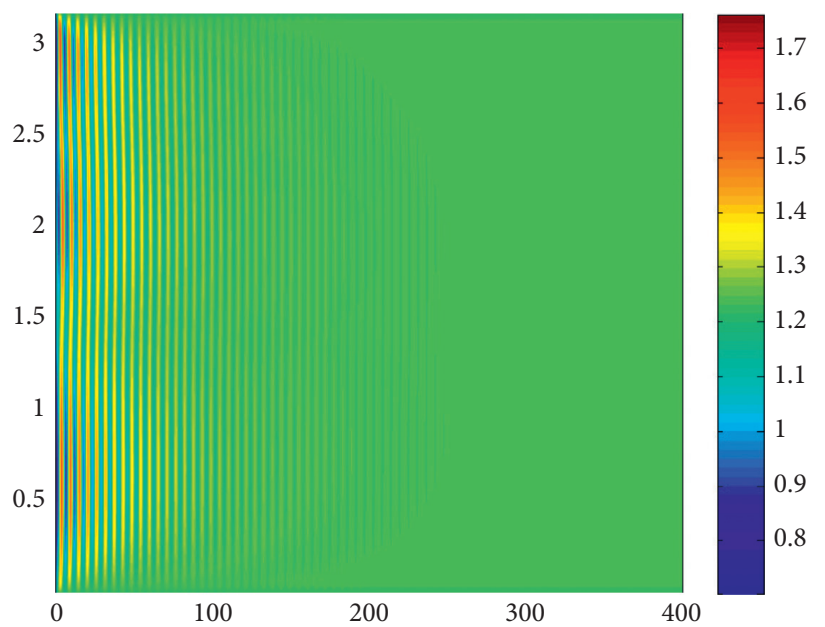

(a)

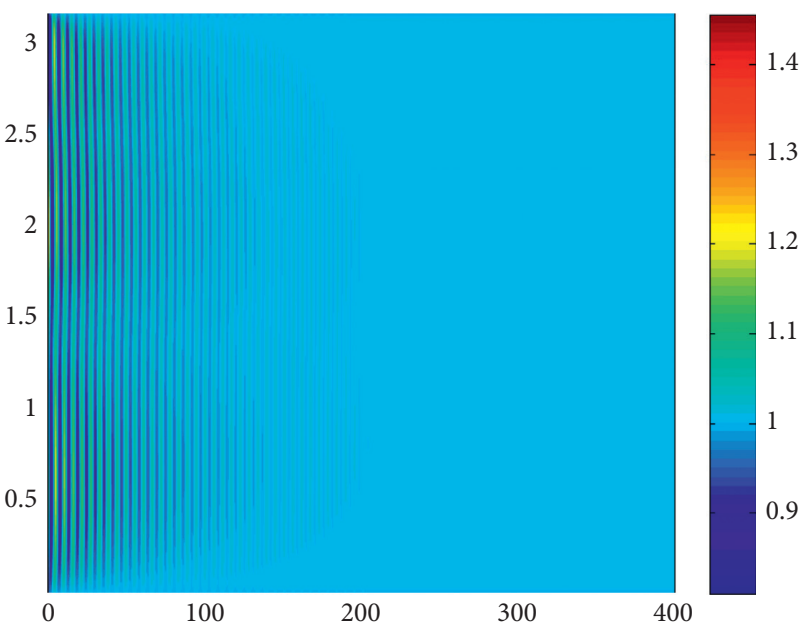

(b)

Figure 6: The spatial pattern of the system with Dirichlet boundary for $\tau_{1}=0.9, \tau_{2}=0.9$, and $\tau=\tau_{1}+\tau_{2}<\tau_{0}^{0}=2.3633$. (a) Spatial pattern of $y_{1}(t, x)$. (b) Spatial pattern of $y_{2}(t, x)$. 


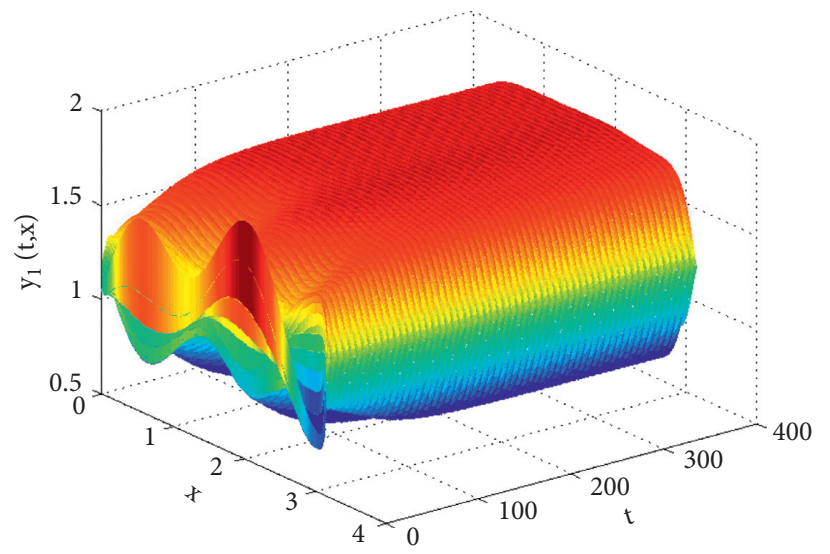

(a)

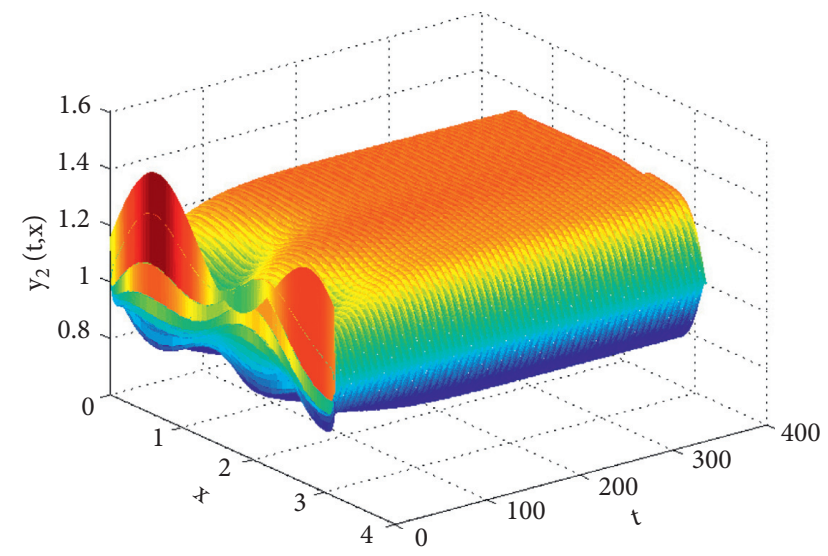

(b)

Figure 7: The positive constant steady state of the system with Dirichlet boundary loses its stability, and Hopf bifurcation occurs for $\tau_{1}=0.9, \tau_{2}=1.5$, and $\tau=\tau_{1}+\tau_{2}>\tau_{0}^{0}=2.3633$. (a) Spatial distribution of $y_{1}(t, x)$. (b) Spatial distribution of $y_{2}(t, x)$.

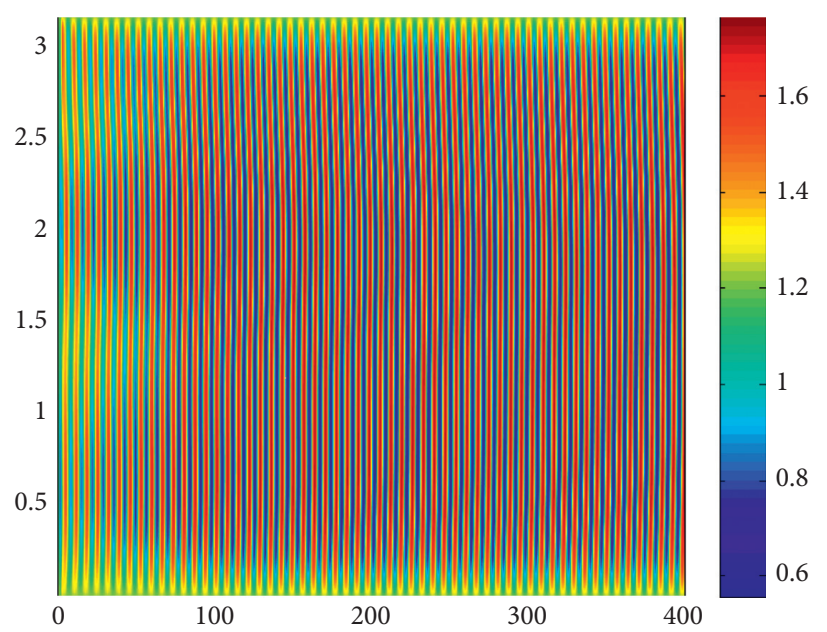

(a)

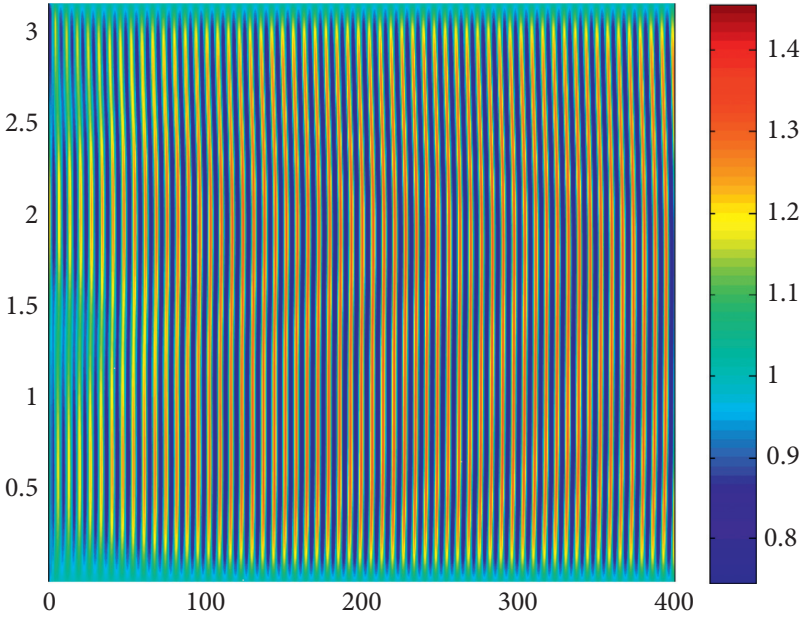

(b)

FIGURE 8: The spatial pattern of the system with Dirichlet boundary for $\tau_{1}=0.9, \tau_{2}=1.5$, and $\tau=\tau_{1}+\tau_{2}>\tau_{0}^{0}=2.3633$. (a) Spatial pattern of $y_{1}(t, x)$. (b) Spatial pattern of $y_{2}(t, x)$.

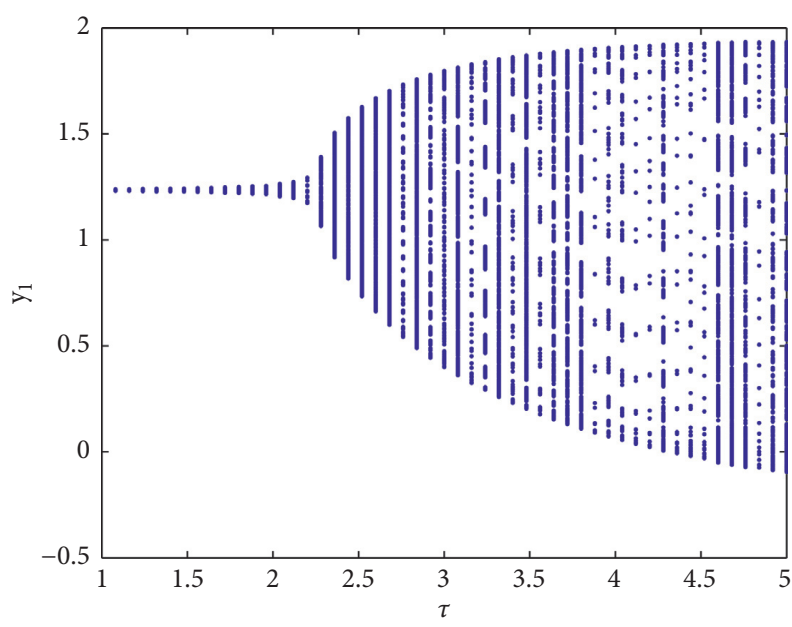

(a)

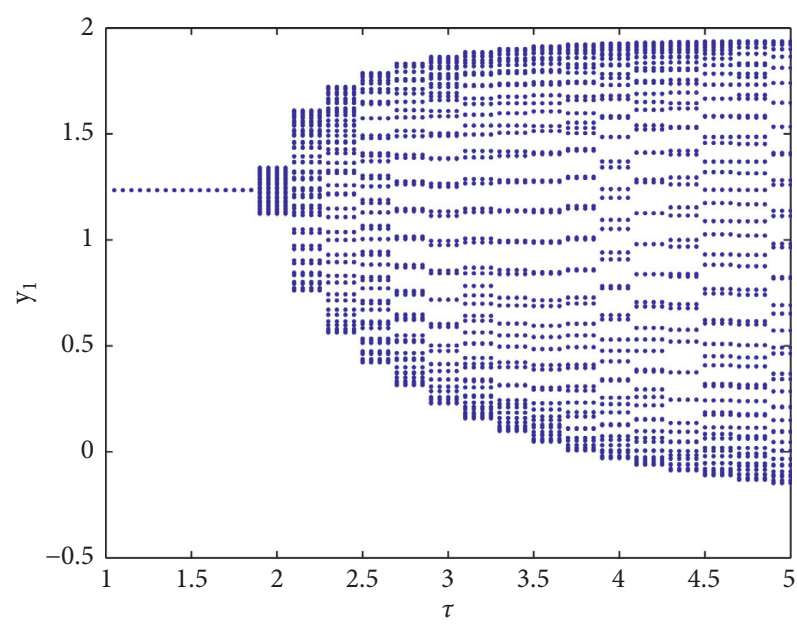

(b)

FIgURE 9: The bifurcation diagram of the system for $y_{1}$ with respect to $\tau$. (a) Without diffusion. (b) With diffusion. 


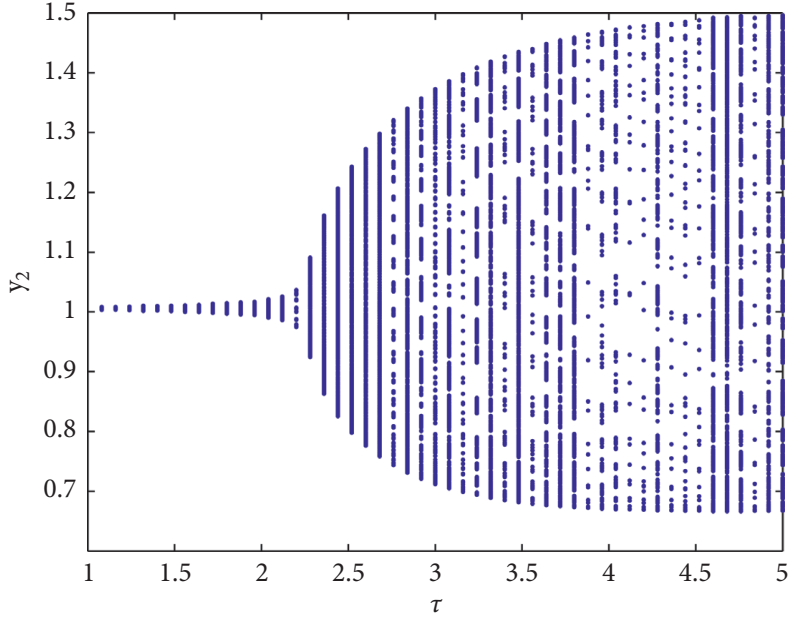

(a)

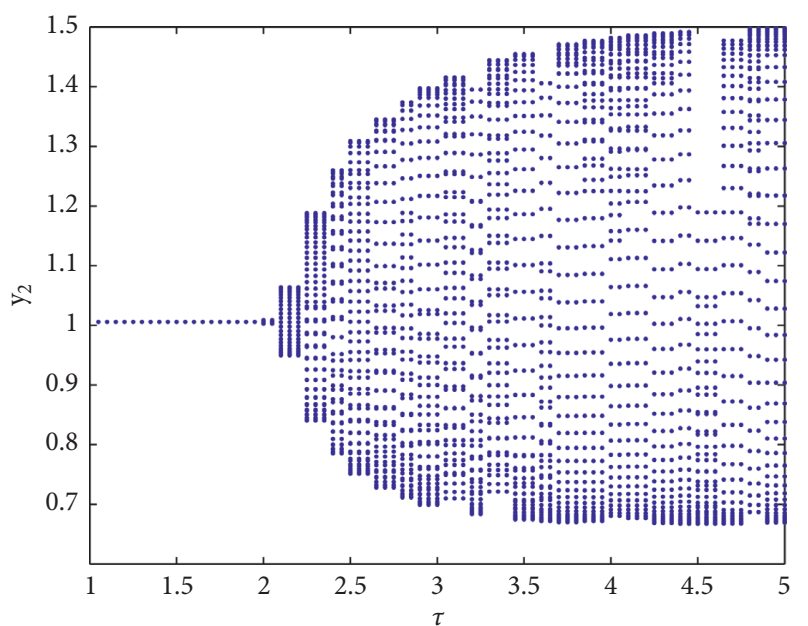

(b)

Figure 10: The bifurcation diagram of the system for $y_{2}$ with respect to $\tau$. (a) Without diffusion. (b) With diffusion.
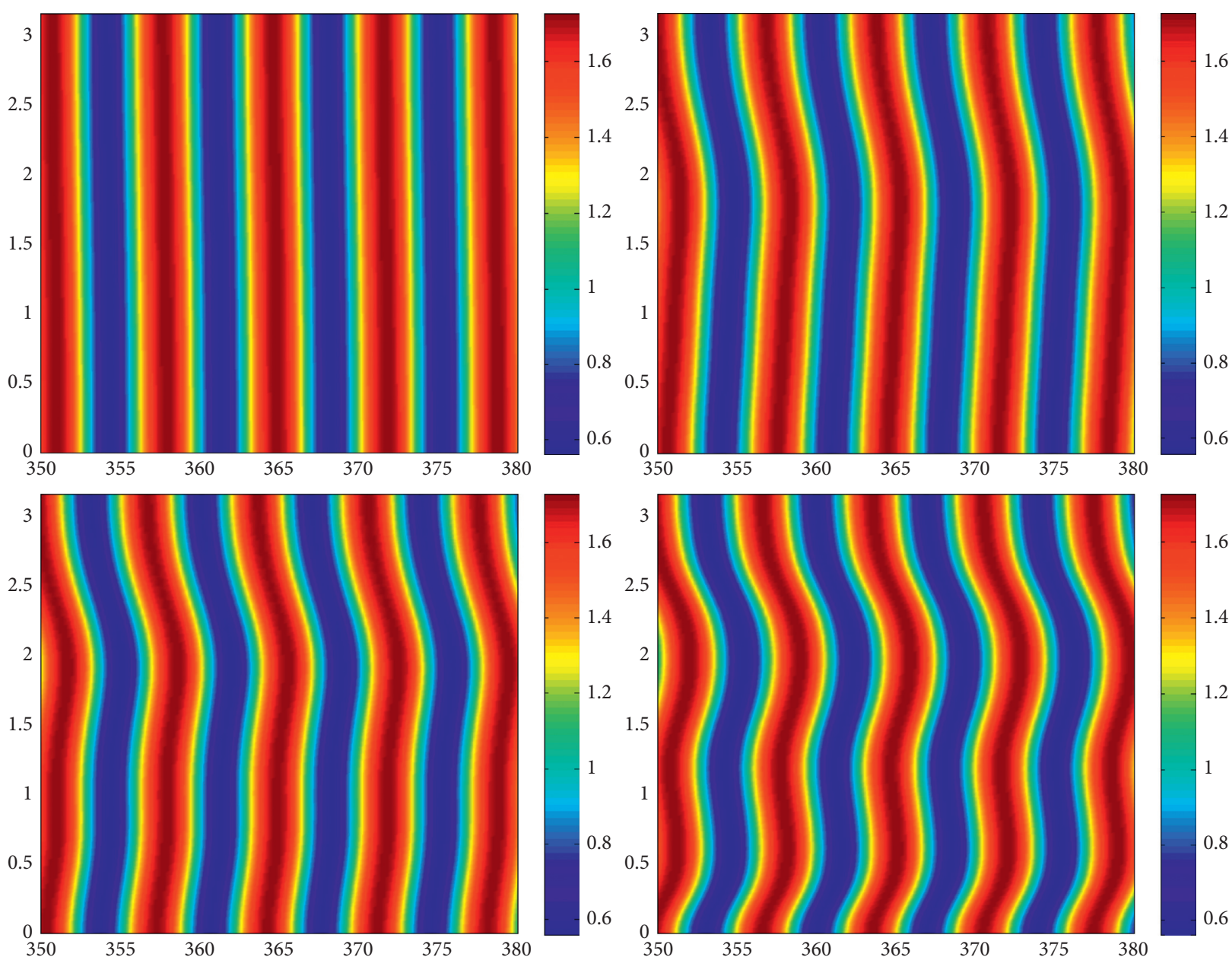

FIGURE 11: The spatial pattern of the system with Neumann boundary for $y_{1}$ with different $D_{i}$ at times $t=350-380$ (from top to bottom, $\left.D_{i}=0.01,0.001,0.0005,0.0001\right)$. 

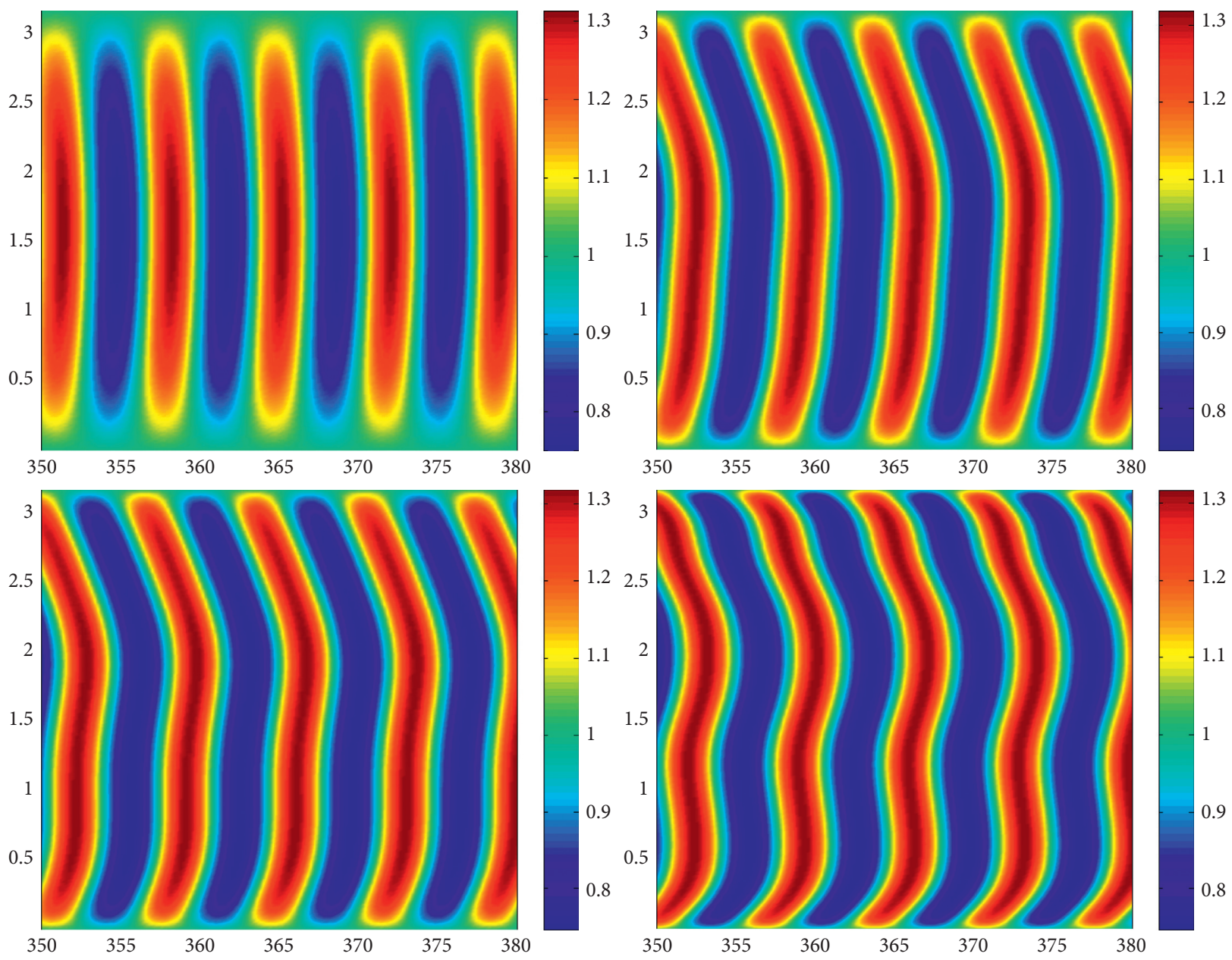

Figure 12: The spatial pattern of the system with Dirichlet boundary for $y_{2}$ with different $D_{i}$ at times $t=350-380$ (from top to bottom, $\left.D_{i}=0.01,0.001,0.0005,0.0001\right)$.

(i) If $\mu_{2}>0\left(\mu_{2}<0\right)$, the direction of the Hopf bifurcation is forward (backward)

(ii) If $\beta_{2}<0\left(\beta_{2}>0\right)$, the bifurcating periodic solutions are stable (unstable)

(iii) If $T_{2}>0\left(T_{2}<0\right)$, the period increases (decreases)

\section{Numerical Simulations}

Example 1. We choose $D_{1}=D_{2}=1, c_{1}=c_{2}=1.5, d_{1}=1$, $d_{2}=0.6, a_{1}=0.4, a_{2}=0.9, b_{1}=0.5$, and $b_{2}=0.2$, which satisfy the above conditions. We take the initial conditions as

$$
\begin{aligned}
& y_{1}(t, x)=1.2354 \times(1+0.2 \sin (3.7 x)+0.3 \sin (1.4 x-0.6)), \\
& y_{2}(t, x)=1.0058 \times(1+0.2 \sin (2.7 x)+0.3 \sin (0.74 x+0.5)) .
\end{aligned}
$$

Then, system (4) with the Neumann boundary has a unique positive constant steady state $E=(1.2354,1.0058)$. By performing calculations, we can obtain $\omega_{+}^{0}=0.8838$ and $\tau_{0}^{0}=2.3633$. According to Theorem $2, E$ is locally asymptotically stable if $\tau \in\left[0, \tau_{0}^{0}\right)$ and is unstable if $\tau>\tau_{0}^{0}$. When $\tau$ crosses through the critical point $\tau_{0}^{0}, E$ loses its stability, and Hopf bifurcation occurs. The simulation results are shown in
Figures 1-4. Based on formulas in Section 3, we can perform computations and obtain that $\operatorname{Re}\left(c_{1}(0)\right)=-2.7368$, $\mu_{2}=1.8499>0$, and $\beta_{2}=-5.4735<0$. Hence, when $\tau$ crosses through $\tau_{0}^{0}$, the bifurcation takes place, and a homogeneous, asymptotically stable periodic solution appears near the trivial steady-state solution $E$, both of which are shown in Figures 3 and 4.

Example 2. In this case, we choose diffusion coefficients $D_{1}=D_{2}=0.001$, and we keep other parameter values and initial conditions unchanged. We consider Hopf bifurcation of the system with the Dirichlet boundary condition:

$$
\begin{aligned}
& y_{1}(t, 0)=y_{1}(t, \pi)=1.2, \\
& y_{2}(t, 0)=y_{2}(t, \pi)=\tanh \left(1+\frac{t}{3 \pi}\right) .
\end{aligned}
$$

The results of the simulation are shown in Figures 5-8. Apparently, when $\tau$ passes through the critical value $\tau_{0}^{0}$, Hopf bifurcation occurs, and the spatially homogeneous bifurcating periodic solution appears, which suggests that there are periodic oscillations in the interaction between two enterprises. 
Figures 9 and 10 show the bifurcation state of the system with and without diffusion for $y_{1}$ and $y_{2}$ under the Neumann boundary, respectively. It is found that the diffusion can affect the amplitude of oscillation of the periodic solutions to a certain extent, and the bifurcating periodic solutions are regular and orbitally asymptotically stable.

To illustrate the spatial effect of diffusion on the system, we adopt the method used by references $[14,21]$ and choose different diffusion coefficients for two different boundary systems under the situations of asymptotically stable periodic solutions. The corresponding spatially dynamic behavior diagrams are shown in Figures 11 and 12.

\section{Conclusions}

Since the diffusions of information, technology, and products exist objectively, we deem that the model with reactiondiffusion and delays is more suitable for the interaction between two enterprises, which has no relevant results for system (3) at present. The aim of this study is first to introduce multiple delays and diffusion factors into the traditional two-enterprise interaction model and then to explore the asymptotic stability of the equilibrium and the periodicity of the solution by taking the sum of two time delays as the bifurcation parameter. It is shown that when the time delay passes through a critical value, the system changes from a stable state to an unstable state. The numerical simulations demonstrate that the diffusion effect causes rich, spatially dynamic behaviors.

We acknowledge that there are some economic implications. In the enterprise cluster, two enterprises can achieve a stable state and symbiosis of development via dynamic interaction. Although there exists competition, cooperation is very effective, and the enterprises can achieve long-term codevelopment through appropriate positive interactions. It is noteworthy that in addition to the varying economic environments, competition powers, mark-saturation degrees, and delay effects, the diffusion speed of products or technologies is an important factor in the self-development of enterprises. When enterprises are in a state of cyclical fluctuations, appropriate diffusion effects may change the amplitude of these fluctuations, which is beneficial to the enterprises to a certain extent. Furthermore, it is easy to find that differences in the initial resources or positions of an enterprise have a certain impact on its future self-development. Therefore, it is obvious that with the development of the digital economy and the emergence of industrial clusters, the internal relationships between enterprises are increasingly in line with the ecological model. As a consequence, it is of great practical significance to study the dynamic properties of these systems for understanding the evolution of market structure and taking corresponding regulatory strategies. Benefiting from the latest research studies, we will further consider the dynamic behaviors of discrete-time and fractional-order models in the future.

\section{Data Availability}

The data used to support the findings of this study are included within the article.

\section{Conflicts of Interest}

The authors declare that they have no conflicts of interest.

\section{Acknowledgments}

This research was supported by the Chongqing Big Data Engineering Laboratory for Children, Chongqing Electronics Engineering Technology Research Center for Interactive Learning, Chongqing University Innovation Research Group, Chongqing Key Discipline of Electronic Information, Chongqing Natural Science Foundation Project (CSTC2021-msxm1993), the program of Chongqing University of Education (KY202116C), and the Science and Technology Research Program of Chongqing Municipal Education Commission (KJQN202101614).

\section{References}

[1] L. Guerrini, "Small delays in a competition and cooperation model of enterprises," Applied Mathematical Sciences, vol. 10, pp. 2571-2574, 2016.

[2] L. Li, C.-H. Zhang, and X.-P. Yan, "Stability and Hopf bifurcation analysis for a two-enterprise interaction model with delays," Communications in Nonlinear Science and Numerical Simulation, vol. 30, no. 1-3, pp. 70-83, 2016.

[3] M. Liao, C. $\mathrm{Xu}$, and $\mathrm{X}$. Tang, "Dynamical behaviors for a competition and cooperation model of enterprises with two delays," Nonlinear Dynamics, vol. 75, no. 1-2, pp. 257-266, 2014.

[4] M. Liao, C. Xu, and X. Tang, "Stability and Hopf bifurcation for a competition and cooperation model of two enterprises with delay," Communications in Nonlinear Science and Numerical Simulation, vol. 19, no. 10, pp. 3845-3856, 2014.

[5] C. J. Xu, "Periodic behavior of competition and corporation dynamical model of two enterprise on time scales," Journal of Quantitative Economics, vol. 29, pp. 1-4, 2012.

[6] C. Xu and P. Li, "Almost periodic solutions for a competition and cooperation model of two enterprises with time-varying delays and feedback controls," Journal of Applied Mathematics and Computing, vol. 53, no. 1-2, pp. 397-411, 2015.

[7] C. Huang, J. Wang, X. Chen, and J. Cao, "Bifurcations in a fractional-order bam neural network with four different delays," Neural Networks, vol. 141, pp. 344-354, 2021.

[8] X. Jiang, X. Chen, T. Huang, and H. Yan, "Bifurcation and control for a predator-prey system with two delays," IEEE Transactions on Circuits and Systems II: Express Briefs, vol. 68, no. 1, pp. 376-380, 2021.

[9] L. Lu, Y. Lian, and C. Li, "Dynamics for a discrete competition and cooperation model of two enterprises with multiple delays and feedback controls," Open Mathematics, vol. 15, no. 1, pp. 218-232, 2017.

[10] Z. Wang, Y. Xie, J. Lu, and Y. Li, "Stability and bifurcation of a delayed generalized fractional-order prey-predator model with interspecific competition," Applied Mathematics and Computation, vol. 347, pp. 360-369, 2019.

[11] C. J. Xu, P. L. Li, Q. M. Xiao, and S. Yuan, "New results on competition and cooperation model of two enterprises with multiple delays and feedback controls," Boundary Value Problems, vol. 36, pp. 1-12, 2019.

[12] C. Xu, M. Liao, and P. Li, "Bifurcation control of a fractionalorder delayed competition and cooperation model of two 
enterprises," Science China Technological Sciences, vol. 62, no. 12, pp. 2130-2143, 2019.

[13] C. J. Xu, M. X. Liao, P. L. Li, and S. Yuan, "New insights on bifurcation in a fractional-order delayed competition and cooperation model of two enterprises," Journal of Applied Analysis and Computation, vol. 11, pp. 1240-1258, 2021.

[14] T. Dong, W. Xu, and X. Liao, "Hopf bifurcation analysis of reaction-diffusion neural oscillator system with excitatory-toinhibitory connection and time delay," Nonlinear Dynamics, vol. 89, no. 4, pp. 2329-2345, 2017.

[15] R. Han and B. Dai, "Hopf bifurcation in a reaction-diffusive two-species model with nonlocal delay effect and general functional response," Chaos, Solitons \& Fractals, vol. 96, pp. 90-109, 2017.

[16] H. Hu, Y. Tan, and J. Huang, "Hopf bifurcation analysis on a delayed reaction-diffusion system modelling the spatial spread of bacterial and viral diseases," Chaos, Solitons of Fractals, vol. 125, pp. 152-162, 2019.

[17] W. Hu, H. Zhao, and T. Dong, "Dynamic analysis for a Kaldor-Kalecki model of business cycle with time delay and diffusion effect," Complexity, vol. 2018, Article ID 1263602, 11 pages, 2018.

[18] J. Huang, Z. Liu, and S. Ruan, "Bifurcation and temporal periodic patterns in a plant-pollinator model with diffusion and time delay effects," Journal of Biological Dynamics, vol. 11, no. 1, pp. 138-159, 2016.

[19] F. Liu, R. Yang, and L. Tang, "Hopf bifurcation in a diffusive predator-prey model with competitive interference," Chaos, Solitons \& Fractals, vol. 120, pp. 250-258, 2019.

[20] Z. L. Shen and J. J. Wei, "Bifurcation analysis in a diffusive mussel-algae model with delay," International Journal of Bifurcation and Chaos, vol. 29, 2019.

[21] Y. L. Song, H. P. Jiang, Q. X. Liu, and Y. Yuan, "Spatiotemporal dynamics of the diffusive Mussel-Algae model near Turing-Hopf bifurcation," SIAM Journal on Applied Dynamical Systems, vol. 16, pp. 2030-2062, 2017.

[22] Y. Tan, C. Huang, B. Sun, and T. Wang, "Dynamics of a class of delayed reaction-diffusion systems with Neumann boundary condition," Journal of Mathematical Analysis and Applications, vol. 458, no. 2, pp. 1115-1130, 2018.

[23] X. Tang and Y. Song, "Stability, Hopf bifurcations and spatial patterns in a delayed diffusive predator-prey model with herd behavior," Applied Mathematics and Computation, vol. 254, pp. 375-391, 2015.

[24] C. Tian and L. Zhang, "Hopf bifurcation analysis in a diffusive food-chain model with time delay," Computers \& Mathematics with Applications, vol. 66, no. 10, pp. 2139-2153, 2013.

[25] G. X. Yang and F. C. Zhang, "Analysis of effects of delays and diffusion on a predator-prey system," Mathematical Problems in Engineering, vol. 2018, Article ID 2104205, 12 pages, 2018.

[26] R. Yang, "Bifurcation analysis of a diffusive predator-prey system with Crowley-Martin functional response and delay," Chaos, Solitons \& Fractals, vol. 95, pp. 131-139, 2017.

[27] W. Zuo and J. Wei, "Stability and Hopf bifurcation in a diffusive predator-prey system with delay effect," Nonlinear Analysis: Real World Applications, vol. 12, no. 4, pp. 1998-2011, 2011.

[28] B. D. Hassard, N. D. Kazarinoff, and Y. H. Wan, Theory and Applications of Hopf Bifurcation, Cambridge University Press, Cambridge, UK, 1981.

[29] T. Faria, "Normal forms and Hopf bifurcation for partial differential equations with delays," Transactions of the American Mathematical Society, vol. 352, no. 5, pp. 2217-2238, 2000.
[30] J. Wu, Theory and Applications of Partial Functional Differential Equations, Springer-Verlag Press, Berlin, Germany, 1996.

[31] J. Hale, Theory of Functional Differential Equations, SpringerVerlag Press, Berlin, Germany, 1977.

[32] Y. Song, M. Han, and Y. Peng, "Stability and Hopf bifurcations in a competitive Lotka-Volterra system with two delays," Chaos, Solitons \& Fractals, vol. 22, no. 5, pp. 1139-1148, 2004.

[33] Y. L. Song and J. J. Wei, "Local Hopf bifurcation and global periodic solutions in a delayed predator-prey system," Journal of Mathmatical Analysis and Applications, vol. 301, pp. 1-21, 2005. 